\title{
Portfolio Choice Beyond the Traditional Approach*
}

\author{
Francisco Peñaranda \\ UPF, Ramon Trias Fargas 25-27, E-08005 Barcelona, Spain. \\ $<$ francisco.penaranda@upf.edu>
}

March 2007

Preliminary draft

\begin{abstract}
This paper surveys asset allocation methods that extend the traditional approach. An important feature of the the traditional approach is that measures the risk and return tradeoff in terms of mean and variance of final wealth. However, there are also other important features that are not always made explicit in terms of investor's wealth, information, and horizon: The investor makes a single portfolio choice based only on the mean and variance of her final financial wealth and she knows the relevant parameters in that computation. First, the paper describes traditional portfolio choice based on four basic assumptions, while the rest of the sections extend those assumptions. Each section will describe the corresponding equilibrium implications in terms of portfolio advice and asset pricing.
\end{abstract}

Keywords: Mean-Variance Analysis, Background Risks, Estimation Error, Expected Utility, Multi-Period Portfolio Choice.

JEL: D81, G11, G12

${ }^{*}$ Part of this survey is based on material of portfolio management courses at BBVA, CEMFI, and University of Alicante. The author thanks Álvaro Elices, Jón Daníelsson, Lorenzo de Cristóbal, and Ángel León. The author especially thanks Enrique Sentana for detailed comments. Financial support from the MEC ref. SEJ2005$02829 /$ ECON is acknowledged. 


\section{Introduction}

Asset allocation, defined as the optimal distribution of investment across broad sets of assets, is probably the most important component of portfolio management as it is stressed by Brinson et al. (1986) among others. This paper is a survey on asset allocation methods that extend the traditional approach. Scherer (2004) is a good reference for many of the topics we will cover and Brandt (2004) is another good reference, with a focus on econometric issues related to portfolio choice.

An important feature of the traditional approach is that measures the risk and return tradeoff in terms of mean and variance of final wealth, i.e. it takes a particular stand on investor's preferences. Mean-variance theory has been widely used both inside and outside financial economics. Finance applications include topics such as portfolio analysis, asset pricing, and performance measurement.

However, there are also other important features of traditional portfolio choice that are not always made explicit in terms of investor's wealth, information, and horizon: The investor makes a single portfolio choice based only on the mean and variance of her final financial wealth and she knows the relevant parameters in that computation. Therefore, we define traditional portfolio choice as based on four assumptions on the investor:

1. Wealth: There are not background risks and all financial assets are tradable (perfectly liquid). Inflation, human capital, liabilities, etc. are not taken into account.

2. Information: Her information set includes all the relevant parameters in the portfolio choice problem. Specifically, she knows the relevant parameters of the joint distribution of asset returns.

3. Preferences: Under the expected utility paradigm, she only cares about the mean and variance of random outcomes, i.e. her preferences are mean-variance. The relevant measure of risk is variance.

4. Horizon: Her investment horizon is one period in the sense that she only trades at the beginning of that period and cares about utility from final wealth. The investor does not take into account investment opportunities in future periods.

This ordering of assumptions represents an increasing complexity in the required extensions 
once that particular assumption is dropped and hence will be the guideline of this survey. Section 2 describes traditional portfolio choice and the rest of the sections extend one of those assumptions while keeping the rest as given. References that extend several assumptions at the same time will also be provided. Each section will describe the corresponding equilibrium implications in terms of portfolio advice and asset pricing. Even though a survey on portfolio choice could naturally skip asset pricing issues, we consider very relevant to describe the feedback between portfolio choice and asset pricing models.

Section 3 extends the first assumption on investor's wealth, while Section 4 extends the second assumption on investor's knowledge of parameters. These two sections still rely on mean-variance preferences, but the next two represent a significant departure. Section 5 extends the third assumption on investor's preferences and Section 6 extends the fourth assumption on investor's horizon.

Obviously, the previous assumptions are not the only ones that are made. First, portfolio choice based on maximizing expected utility is a maintained hypothesis in this paper. The expected utility paradigm is the canonical model of preferences under uncertainty and plays a central role in portfolio choice theory. The interested reader can find references outside this paradigm in Section 2.4.1 of Brandt (2004). We will also briefly refer to robust portfolio choice in Section 4.3.

We also assume perfect markets in the sense of price-taking behaviour and lack of market frictions such as transaction costs, trading constraints, taxes, etc. Some market frictions can be easily included in the analysis as problem constraints, but this survey is not focused on portfolio choice under different types of constraints for a given (mean-variance) criterion. For instance, we will not add value at risk or probability of shortfall constraints. Nevertheless, we dedicate Section 5 to preferences that are not mean-variance, where we can introduce those constraints as the portfolio choice criterion itself. In addition, Section 4 comments briefly the introduction of constraints to alleviate the effects of estimation error.

There are some topics such as security selection, and hedging and arbitrage strategies, that fall outside the scope of this survey. We will not study security selection because it does not belong to asset allocation. On the other hand, this should not be confused with an emphasis on passive management since we will study market timing (tactical asset allocation) in Section 6.2 and each section will comment the corresponding portfolio advice in terms of holding or not 
the market portfolio. Even though we do not focus on exploiting superior information, Section 3.3 describes Black-Litterman's optimal combination of investor and market views. We will not study portfolio insurance but we will study particular types of hedging induced by portfolio construction in set-ups with background risks and/or time-varying investment opportunities, analyzed in Section 2.2 and 5.2 respectively.

On the other hand, we will comment some topics that might not be regarded as asset allocation issues. We will study benchmark-relative management in Section 2.3 as a simple application of portfolio choice under background risks. We will also study some non-traditional performance measures in Section 4.3 as natural implications of preferences that are not mean-variance.

Practitioners tend lately to focus on the concept of risk budgeting but asset allocation is still relevant in that context. We share the view of Scherer (2004) that portfolio construction and risk budgeting are the same concept, looking for a portfolio that delivers an optimal risk-return trade-off. Portfolio construction is expressed in terms of portfolio weights while risk budgeting uses risk exposures (e.g. VaR), but it is simply a difference in the presentation.

\section{Traditional Portfolio Choice}

\subsection{Asset Allocation Set-Up}

Let us first describe the investment opportunities and the required notation. The gross return of a particular asset (payoff divided by price) is denoted by $R$ and the corresponding rate of return by $r=R-1$. We assume the investor has access to $1 \leq N<\infty$ risky assets. Those assets should be understood as asset classes, e.g. stocks and bonds. In a global asset allocation set-up, we would include currencies, stocks and bonds from different countries or regions. To simplify the exposition, we assume cash (short-term money-market instruments) is another available asset class and plays the role of a safe asset. The risk-free rate of return is denoted by $r_{0}$ and an excess return by $e=r-r_{0}$.

The corresponding vectors of rates of returns and excess returns of risky assets are denoted by $\mathbf{r}$ and $\mathbf{e}$. The corresponding $N \times 1$ mean vectors and $N \times N$ variance-covariance matrix, which we assume well-defined, are

$$
\boldsymbol{\nu}=E(\mathbf{r}), \quad \boldsymbol{\mu}=E(\mathbf{e}), \quad \boldsymbol{\Sigma}=\operatorname{Var}(\mathbf{r})=\operatorname{Var}(\mathbf{e}),
$$

where $\boldsymbol{\mu}$ is known as the vector of risk premia. We make two simplifying assumptions on those 
objects, not all expected returns are equal to the riskless return $(\boldsymbol{\mu} \neq \mathbf{0})$, and $\boldsymbol{\Sigma}$ is nonsingular $(|\boldsymbol{\Sigma}|>0)$. The former assumption is required to define a proper risk-return trade-off and the latter one is required to avoid a "hidden" safe asset, i.e. a non-trivial portfolio with zero variance. We also define $H=\boldsymbol{\mu}^{\prime} \boldsymbol{\Sigma}^{-1} \boldsymbol{\mu}$.

The payoffs that an investor can get are given by portfolios of those assets and financial investment is modelled as a technology with constant returns to scale. Let us define $\mathbf{1}$ as an $N \times 1$ vector of ones and denote the amount of wealth invested in each risky asset class by an $N \times 1$ vector $\mathbf{w}$. Hence $\mathbf{w}^{\prime} \mathbf{1}$ represents the wealth invested in risky assets and the rest of wealth is invested in the safe asset. If we normalize initial wealth to 1 then $1-\mathbf{w}^{\prime} \mathbf{1}$ is the wealth invested in the safe asset. Moreover, we can interpret $\mathbf{w}$ as the proportions of wealth invested in risky asset classes.

We can express the rate of return of a unit-cost portfolio as

$$
r=\mathbf{w}^{\prime} \mathbf{r}+\left(1-\mathbf{w}^{\prime} \mathbf{1}\right) r_{0}=r_{0}+\mathbf{w}^{\prime} \mathbf{e},
$$

and the corresponding notation for its moments is

$$
\begin{gathered}
E(r)=r_{0}+\mathbf{w}^{\prime} \boldsymbol{\mu}=\nu, \quad E(e)=\mathbf{w}^{\prime} \boldsymbol{\mu}=\mu, \\
\operatorname{Var}(r)=\operatorname{Var}(e)=\mathbf{w}^{\prime} \mathbf{\Sigma} \mathbf{w}=\sigma^{2}
\end{gathered}
$$

An investor's portfolio choice maximizes expected utility. Here we impose assumptions 1 and 4. The latter assumption defines final period wealth as the object of interest for the investor and the former one allows the focus on financial wealth, i.e. the payoff of the chosen portfolio. Since we normalize initial wealth to 1 , her final wealth is equal to her portfolio's gross return $R$. The portfolio choice problem is simply

$$
\max _{\mathbf{w}} E[u(R)]
$$

for some von Neumann-Morgenstern (vN-M) utility function ${ }^{1} u(\cdot)$, which is usually strictly increasing and concave to reflect an agent that prefers more to less and shows risk aversion.

\subsection{Mean-Variance Portfolio Choice}

Now we impose assumption 3, which means that we can represent investor's expected utility as a function of her return's mean and variance $E[u(R)]=U\left(\nu, \sigma^{2}\right)$, where $\left(\nu, \sigma^{2}\right)$ are given

\footnotetext{
${ }^{1}$ We follow the usual convention in finance references. Other references might use the concept of vN-M utility for $E[u(R)]$ instead.
} 
in (2) and the function $U(\cdot)$ is such that $\partial U / \partial \nu>0, \partial U / \partial \sigma<0$, and $U\left(\nu, \sigma^{2}\right)$ is (strictly) concave. Variance is the risk measure in this set-up. It is widely used in finance since it delivers simple expressions and rich empirical implications, even though that it is not necessarily an accurate measure of risk. Portfolio choice under mean-variance preferences was developed in Markowitz $(1952,1959)$ and Tobin (1958).

A mean-variance investor solves a special version of the problem (3)

$$
\max _{\mathbf{w}} U\left(\nu, \sigma^{2}\right)
$$

and we can study her indifference curves on $\left(\nu, \sigma^{2}\right)$ or $(\nu, \sigma)$ spaces, where $\nu$ is usually drawn on the y-axis. ${ }^{2}$ We will focus on the space $(\mu, \sigma)$ from now on, which simply changes the origin on the y-axis. She will choose the tangency between her indifference curves and the meanvariance frontier $\sigma^{2}(\mu)$, which represents the best mean-variance trade-off that investors can get from available assets. See Figure 1, where the dotted curves are indifference curves, the arrow shows the direction of higher expected utility, and the mean-variance frontier is the solid line. Therefore, we turn to study $\sigma^{2}(\mu)$.

The mean-variance optimization frontier can be represented in three equivalent ways: Minimizing variance for each target risk premia $\mu$ to emphasize portfolio diversification

$$
\min _{\mathbf{w}} \operatorname{Var}(e) \quad \text { s.t. } \quad E(e)=\mu,
$$

where the dependence of $\operatorname{Var}(e)$ and $E(e)$ on $\mathbf{w}$ is given by (2), maximizing risk premium for each target variance $\sigma^{2}$ in the spirit of risk budgeting

$$
\max _{\mathbf{w}} E(e) \quad \text { s.t. } \quad \operatorname{Var}(e)=\sigma^{2},
$$

or maximizing a trade-off between risk and return given by each risk tolerance $\lambda$

$$
\max _{\mathbf{w}} E(e)-\frac{1}{2 \lambda} \operatorname{Var}(e) \text {. }
$$

By choosing the corresponding $\mu, \sigma^{2}$ and $\lambda$, we can make the three solutions equal. The corresponding algebra for a general $N$ was developed explicitly by Merton (1972). A textbook

\footnotetext{
${ }^{2}$ We can be more precise with a classical example: vN-M exponential (or CARA) utility $u(R)=-\exp (-\theta R)$, where $\theta>0$ is the coefficient of absolute risk aversion, plus normally distributed $R \sim N\left(1+v, \sigma^{2}\right)$. In that context,

$$
E[-\exp (-\theta R)]=-\exp \left[-\theta(1+v)+\frac{1}{2} \theta^{2} \sigma^{2}\right]
$$

which is an ordinal utility function of $\left(v, \sigma^{2}\right)$ and can also be represented by $v-0.5 \theta \sigma^{2}$.
} 
treatment can be found in Chapter 3 of Huang and Litzenberger (1988) and Chapter 4 of Ingersoll (1987). The case of an unbounded $N$ was studied by Chamberlain and Rothschild (1983). They embed mean-variance analysis into Hilbert space theory.

The risky component of the optimal portfolio is

$$
\mathbf{w}(\mu)=\left(\frac{\mu}{H}\right) \mathbf{\Sigma}^{-1} \boldsymbol{\mu},
$$

in the first representation, while it is $\mathbf{w}(\lambda)=\lambda \boldsymbol{\Sigma}^{-1} \boldsymbol{\mu}$ in the last representation. Stevens (1998) gives an insightful representation of the matrix $\boldsymbol{\Sigma}^{-1}$, and hence the vector $\boldsymbol{\Sigma}^{-1} \boldsymbol{\mu}$, in terms of regression hedges. Think of a least squares regression of a particular excess return on a constant and the rest of excess returns. That excess return's entry in $\boldsymbol{\Sigma}^{-1} \boldsymbol{\mu}$ is equal to the ratio of the constant over the residual variance of that regression.

Let us focus on the solution of the first representation. The main property of $\mathbf{w}(\mu)$ is its proportionality to $\boldsymbol{\Sigma}^{-1} \boldsymbol{\mu}$, i.e. the target $\mu$ only rescales the previous vector and relative weights in the optimal risky position are constant across the frontier. In fact, we can compute any optimal portfolio from any pair of optimal portfolios, which is known as two-fund spanning. However, the natural choice is the safe asset and the tangency portfolio, a portfolio of risky assets that the mean-variance frontiers with and without risky assets share. See Figure 2, where the dotted curve represents the mean-variance frontier without a safe asset. The interested reader can find the details about that frontier and the characterization of such tangency in the references.

The mean-variance frontier is the variance corresponding to the optimal portfolio (5) for each target $\mu$

$$
\sigma^{2}(\mu)=\mathbf{w}(\mu)^{\prime} \mathbf{\Sigma} \mathbf{w}(\mu)=\frac{1}{H} \mu^{2} .
$$

The global minimum variance portfolio is simply the safe asset. The frontier is composed by two straight lines on the space $(\mu, \sigma)$, where it is usually drawn with $\mu$ on the y-axis. Of course, we can also draw it on the $\left(\mu, \sigma^{2}\right)$ space, where it becomes a parabola.

Mean-variance efficient portfolios are defined as those portfolios that are not dominated in mean-variance space by any other portfolio. Therefore, (6) shows that the efficient frontier is given by portfolios (5) with $\mu \geq 0$. This part of the frontier is the solid line in Figure 1 and 2. Mean-variance investors' portfolios will be located on the efficient frontier and this (meanvariance) efficient set of portfolios is convex. In addition, we find a trade-off between risk and 
return on the efficient side of the frontier, we can only target a higher $\mu$ if we are willing to suffer a higher $\sigma^{2}$.

\subsection{Equilibrium Implications}

We have not been very explicit about mean-variance parameters so far. Now we impose assumption 2 and hence all investors use the same mean-variance parameters and they are equal to the true ones. We will think of a set of investors, each of them with some mean-variance preferences $U\left(v, \sigma^{2}\right)$ and some initial wealth.

In this context, we will study the equilibrium implications of the previous set-up in terms of portfolio advice and asset pricing. This theory is called the Capital asset pricing model (CAPM) and was developed by Sharpe (1964), Lintner (1965), and Mossin (1966). More recently, Berk (1997) develops the necessary and sufficient conditions for the CAPM under expected utility. We will focus on the CAPM with a safe asset, the reader can find a CAPM without it in Black (1972). The CAPM provides a precise link of risk and return and hence it is widely applied even though it does not hold empirically as described in Chapter 5 in Campbell et al. (1997) and Chapter 20 in Cochrane (2001).

Let us define the market portfolio as the aggregate supply of risky assets, with weights given by market capitalization. It includes all risky assets in the economy since we assume everything is traded through assumption 1, but the market is understood as listed equities (the stock market) in the usual applications. Under equilibrium, the market portfolio must be equal to the aggregation of agents' demands, which are mean-variance efficient. The convexity of the mean-variance efficient set implies that the market portfolio must be efficient, which is the basic implication of the CAPM. The efficient part of the linear frontier is called the Capital market line (CML). Moreover, if we assume a zero net supply of the riskless asset then the market portfolio is equal to the tangency portfolio.

By two-fund spanning, we can represent every investor's portfolio as a combination of the safe asset and the market portfolio and this type of situation is labelled as two-fund separation. Being more (less) risk averse simply translates into investing less (more) on the market portfolio. Therefore, the portfolio advice of the CAPM is passive investment, i.e. investors should hold the market portfolio. This implication of the CAPM had a big impact in the industry, spurring the use of indexed funds. 
In the real world, not every agent invests in the same portfolio of risky assets. Professional advice recommends a higher ratio of stocks with respect to bonds the higher the aggressiveness and the investment horizon of the investor as Canner et al. (1997) point out. They also stress that the absence of a riskless asset cannot rationalize this advice because the mix of bonds and stocks would change with risk aversion, but not in the same direction as professional advice. Short-sale constraints on cash positions cannot rationalize the advice either. Moreover, given the historical low Sharpe ratio of bonds compared to stocks, it looks like mean-variance investors should not invest much in bonds. Next sections will show how the theoretical advice gets closer to real world portfolios once we drop some of the traditional four assumptions.

Another big impact of mean-variance analysis in the investment industry has been the development of certain performance measures. One of such measures is the Sharpe ratio (others examples are the Treynor index and the Jensen's alpha), which is defined as a risky portfolio's risk premium per unit of risk

$$
S R=\frac{\mu}{\sigma}
$$

and was developed in Sharpe (1966) to evaluate mutual funds following the implications of mean-variance analysis. Obviously, we can compare Sharpe ratios of different portfolios without relying on an equilibrium set-up, but this measure is meaningful when we think of the CML. Under mean-variance preferences, there is an optimal benchmark for every agent, the slope of the CML on the $(\mu, \sigma)$ space $(\sqrt{H})$. Sharpe (1994) reviewed the literature that this measure generated and clarified its application.

Finally, let us briefly comment the asset pricing implications of the CAPM. In equilibrium, any risky portfolio with excess return $e$ and risk premium $\mu$ must satisfy

$$
\mu=\beta \mu_{M}, \quad \beta=\frac{\operatorname{Cov}\left(e, e_{M}\right)}{\operatorname{Var}\left(e_{M}\right)},
$$

where $e_{M}$ is the market excess return and $\mu_{M}$ is the market risk premium. Every risk premium is equal to the corresponding beta, the risk measure in the CAPM, times the market risk premium, and this linear relationship is called the Security market line (SML).

Unfortunately, the pricing equation (8) is simply a direct implication of the mean-variance frontier algebra, i.e. it is true if we substitute the market portfolio for any frontier portfolio. Moreover, the market portfolio is unobservable and researchers usually work with a stock index as a proxy. Therefore, testing the CAPM is not a straightforward application of (8) as Roll (1977) stressed. 


\section{Background Risks}

\subsection{New Framework}

Assumption 1 stated that there are not background risks and all financial assets are tradable or perfectly liquid. Obviously, not every risk or asset can be traded and hence a more realistic set-up is portfolio choice of risky securities (endogenous risks) while facing exogenous and unavoidable background risks.

Let us study a simple way of introducing the latter risks in the portfolio construction. Instead of managing the risk-return trade-off of $r$, we manage $a=r-b$, where $b$ is the background risk and covers several situations such as:

- Inflation: $b$ is the relevant inflation rate and $a$ the corresponding real returns. This case was studied by Friend et al. (1976) and Solnik (1978).

- Human capital: $b$ is minus the return on human capital, which is treated as uninsurable. This case was studied by Mayers (1973) and Brito (1977) as the most important example of a nontradable asset. Friend et al. (1976) also analyze the joint effect of inflation and human capital.

- Liabilities: In the context of asset-liability management (ALM) of a pension fund, Sharpe and Tint (1990) define surplus returns as final surplus $S_{1}=A_{1}-L_{1}$ (the difference between assets and liabilities) relative to initial assets $A_{0}$. They can be expressed as $S_{1} / A_{0}=R-\left(L_{0} / A_{0}\right)\left(L_{1} / L_{0}\right)=R-b$, where $L_{1} / L_{0}$ is the source of background risk.

Portfolio returns are still given by (1) but the relevant mean and variance are now

$$
\begin{gathered}
E(a)=E(r)-E(b)=\delta, \\
\operatorname{Var}(a)=\operatorname{Var}(r)+\operatorname{Var}(b)-2 \operatorname{Cov}(r, b)=\omega^{2} .
\end{gathered}
$$

Note that cash is not riskless if our risk measure is $\omega^{2}$ instead of $\sigma^{2}$. The new key object is the covariance of risky securities with background risk

$$
\gamma=\operatorname{Cov}(\mathbf{r}, b)
$$

and the notation $F=\gamma^{\prime} \boldsymbol{\Sigma}^{-1} \boldsymbol{\mu}$ will be used in some expressions. 
The previous examples might also require an explicit modelling of long-term or dynamic portfolio choice, i.e. relax assumption 4 at the same time. The corresponding references will be given in Section 6.4.

\subsection{Background Risks in a Mean-Variance Framework}

This section follows Mayers (1973), Brito (1977), and mainly Solnik (1978). We will focus on the case of a safe asset and the reader can find the case without a safe asset in those references. The optimal portfolio is now given by

$$
\min _{\mathbf{w}} \operatorname{Var}(a) \quad \text { s.t. } \quad E(a)=\delta,
$$

where $\operatorname{Var}(a)$ and $E(a)$ are given by (9), which can be equivalently expressed as

$$
\min _{\mathbf{w}} \operatorname{Var}(e)-2 \operatorname{Cov}(e, b) \quad \text { s.t. } \quad E(e)=\mu
$$

and the only difference with the traditional problem (4) is the component $\operatorname{Cov}(e, b)=\mathbf{w}^{\prime} \boldsymbol{\gamma}$ in the risk criterion. We skipped the component $\operatorname{Var}(b)$ in $\operatorname{Var}(a)$ and $E(b)$ in $E(a)$ because they are not affected by the portfolio choice. The solution can be represented as

$$
\mathbf{w}_{b}(\mu)=\mathbf{w}(\mu)+\mathbf{w}_{h},
$$

the traditional optimal portfolio for $\mu$ in (5) plus a constant term, a hedging demand due to background risk,

$$
\mathbf{w}_{h}=\boldsymbol{\Sigma}^{-1} \boldsymbol{\gamma}-\left(\frac{F}{H}\right) \boldsymbol{\Sigma}^{-1} \boldsymbol{\mu} .
$$

The component $\boldsymbol{\Sigma}^{-1} \boldsymbol{\gamma}$ can be interpreted as the coefficients of $\mathbf{r}$ in the least squares regression of $b$ on a constant and the vector $\mathbf{r}$. Let us think of a diagonal $\boldsymbol{\Sigma}$ to fix ideas. Then a higher entry in $\gamma$ increases the demand for that asset since it is a hedging instrument, paying relatively more when the background risk is relatively higher. For instance, if $b$ is similar to bonds then this effect might motivate some investment in bonds that would be missing in mean-variance analysis applied to historical data, where bonds' Sharpe ratios are low.

Now the target $\mu$ is not the only object that might differ across investors. The hedging demand $\mathbf{w}_{h}$ might depend on the investor since the relevant $b$ might do. There is two-fund spanning of each investor's frontier, but none of the two funds is the safe asset in general. Now the relative weights of risky assets might change with the target $\mu$ or investor's risk aversion. 
The background risk frontier is a hyperbola on the $(\delta, \omega)$ space, but that space might depend on the investor. It is more interesting to compare background risk and traditional frontiers on the $(\mu, \sigma)$ space, where the latter is the efficient one. The representation in (10) is very convenient for that purpose. In terms of rates of return, $\mathbf{r}^{\prime} \mathbf{w}_{b}(\mu)=\mathbf{r}^{\prime} \mathbf{w}(\mu)+\mathbf{r}^{\prime} \mathbf{w}_{h}$ or simply $r_{b}=r+r_{h}$. The key property is that $r_{h}$ is independent of chosen $\mu$, has a zero mean $\left(E\left(r_{h}\right)=0\right)$, and is orthogonal to $r\left(\operatorname{Cov}\left(r, r_{h}\right)=0\right)$.

We find the decomposition

$$
\operatorname{Var}\left(r_{b}\right)=\operatorname{Var}(r)+\operatorname{Var}\left(r_{h}\right)
$$

and hence the background risk frontier represents a parallel parabola with respect to the traditional frontier on the $\left(\mu, \sigma^{2}\right)$ space, the size of the parallel movement to the right depends on $\operatorname{Var}\left(r_{h}\right)$. The background risk frontier represents a hyperbola with asymptotes equal to traditional lines in the $(\mu, \sigma)$ space. See Figure 3, where the arrow shows the direction of higher hedging demand variance.

The background risk and traditional frontiers coincide $\left(\mathbf{w}_{h}=\mathbf{0}\right)$ when the covariance of assets with background risks $\boldsymbol{\gamma}$ is proportional to $\boldsymbol{\mu}$ since then there is no conflict between the meanvariance and hedging motives. One special case of that situation is a zero covariance $\gamma$ between assets and background risks. Another special case is the existence of a perfect background risk mimicking portfolio $\left(b=r_{0}+\mathbf{w}_{*}^{\prime} \mathbf{e}\right.$ for some $\left.\mathbf{w}_{*}\right)$ that lies on the traditional frontier.

Now the equilibrium implications will be briefly described. In terms of portfolio advice, the tight link between the market portfolio and the individual portfolios is broken. If $b$ is common across agents then we have two-fund separation and the market portfolio might be chosen as one of the funds, but this does not mean a constant mix of risky assets on the frontier. Moreover, a common $b$ will not be the general case and two-fund separation clearly breaks down with human capital. Passive management in the sense of holding the market is not the portfolio advice in this set-up.

We should not expect the market portfolio to be mean-variance efficient even in the case of a common $b$ and hence the CAPM equation (8) breaks down. However, given the structure of (10), if we substract the (average) hedging component from the market portfolio then we have a mean-variance efficient portfolio and hence a properly adjusted market return works in (8). 


\subsection{Application to Tracking Error Optimization}

There is another relevant type of background risk apart from the examples mentioned in Section 3.1. Benchmark-relative investment management is a key ingredient of active portfolio management (i.e. security selection and market timing) as shown in Grinold and Kahn (1999) and Lee (2001). The investment industry usually measures performance relative to a benchmark and a manager might care about her relative performance more than total risk and return. This particular application of background risks might fall outside asset allocation if we think of an active manager given an asset class as her benchmark, but it is also relevant for practical tactical asset allocation as we will comment in Section 6.1.

This section will describe the results of Roll (1992) on tracking error optimization translated to the case of a safe asset. We can easily study this situation in our set-up by interpreting $b=r_{0}+\mathbf{w}_{*}^{\prime} \mathbf{e}$ as the benchmark, and referring to $a$ as active returns

$$
a=r-b=\left(\mathbf{w}-\mathbf{w}_{*}\right)^{\prime} \mathbf{e}=\mathbf{w}_{a}^{\prime} \mathbf{e} .
$$

and $\omega$ as tracking error (TE)

$$
\operatorname{Var}(a)=\mathbf{w}_{a}^{\prime} \mathbf{\Sigma} \mathbf{w}_{a}=\omega^{2},
$$

which is a widely used measure of relative investment risk. We will also use the notation $E\left(b-r_{0}\right)=\mu_{*}$ and $\operatorname{Var}\left(b-r_{0}\right)=\sigma_{*}^{2}$.

Now we focus on the active portfolio instead of the total portfolio, but the problem is still the same (a self-financing constraint should be added if there was not a safe asset),

$$
\min _{\mathbf{w}_{a}} \operatorname{Var}(a) \quad \text { s.t. } \quad E(a)=\delta,
$$

and the optimal active portfolio can be represented as $\mathbf{w}_{a}(\delta)=(\delta / H) \boldsymbol{\Sigma}^{-1} \mu$. A noteworthy feature of the optimal active portfolio is its independence from the particular benchmark. It is not difficult to relate (10) to the current representation

$$
\mathbf{w}_{b}(\mu)=\mathbf{w}_{a}(\delta)+\mathbf{w}_{*}=\left[\mathbf{w}_{a}(\delta)+\mathbf{w}\left(\mu_{*}\right)\right]+\left[\mathbf{w}_{*}-\mathbf{w}\left(\mu_{*}\right)\right]=\mathbf{w}(\mu)+\mathbf{w}_{h} .
$$

The role of the target $\delta$ is simply to scale a vector and the efficient part of the TE frontier on space $(\delta, \omega)$ is a straight line that starts at the origin. Again, it is more interesting to face the TE frontier against the traditional frontier on space $\left(\mu, \sigma^{2}\right)$, i.e. the mean and variance of $r_{b}=a+b$ in excess of $r_{0}$. Given the previous representation of optimal TE portfolios,

$$
\operatorname{Var}\left(r_{b}\right)=\sigma^{2}(\mu)+\left[\sigma_{*}^{2}-\sigma^{2}\left(\mu_{*}\right)\right]
$$


where $\sigma^{2}(\cdot)$ is the mean-variance frontier (6) evaluated at a particular target. The TE frontier represents a parallel parabola on the $\left(\mu, \sigma^{2}\right)$ space, it passes through the benchmark and is dominated at all return levels by mean-variance frontier. The distance between both frontiers is equal to the benchmark inefficiency.

TE optimization might be detrimental to overall portfolio efficiency. On the other hand, if $b$ is efficient then both frontiers coincide and TE efficient portfolios are mean-variance efficient. Roll (1992) also comments the introduction of a beta (sensitivity with respect to the benchmark) constraint to decrease the inefficiency of the TE portfolios on the total mean-variance space.

\section{Parameter Uncertainty}

\subsection{Estimation Error in Mean-Variance Inputs}

Assumption 2 states that investors know the true mean $\boldsymbol{\mu}$ and variance $\boldsymbol{\Sigma}$ required in the portfolio choice problem. Obviously, those inputs are uncertain and it is common to simply plug in (5) historical estimates of those parameters by means of a time series of size $T$ of the relevant returns. ${ }^{3}$ Then well-known problems of traditional mean-variance analysis show up: Extreme long/short positions and unstable portfolios, in the sense that a slight change in the target gives significant portfolio changes. Best and Grauer (1991) study the sensitivity in mean-variance portfolios' weights and moments to changes in the means.

Finding badly behaved portfolios when using sample estimates as inputs raises concerns about estimation error in mean-variance inputs. Michaud (1989) stresses that portfolio optimization based on historical estimates suffers from error maximization. Optimal portfolios take extreme positions that are mainly driven by estimation error, e.g. extremely long in assets with overestimated returns and/or underestimated risk, and hence they perform poorly out of sample. Chopra and Ziemba (1993) study the effect of errors in mean and variance in portfolios and conclude that the former errors are more important.

There is a difference in the relative magnitude of estimation errors. The variance of returns can be estimated more accurately than their mean if returns follow diffusions as Merton (1980) shows. If we try to estimate the mean and the variance of log-returns by means of their sample counterparts then the standard error of the mean estimator decreases with the data span (say

\footnotetext{
${ }^{3}$ If one takes seriously the CAPM this is not necessary because the investor should simply hold the market portfolio. This will be exploited in Section 4.3 with the Black-Litterman approach.
} 
the number of years), while the standard error of the variance estimator decreases with the number of observations. For instance, stock returns are very noisy and an accurate estimation of risk premia would require many years of data, while the estimation of variances can be made arbitrarily accurate by increasing the data frequency. ${ }^{4}$

The simplest approach to avoid unreasonable mean-variance portfolios with historical estimates is to impose constraints in the mean-variance optimization (4), e.g. lineal constraints

$$
\max _{\mathbf{w}} E(e)-\frac{1}{2 \lambda} \operatorname{Var}(e) \quad \text { s.t. } \quad \mathbf{a}_{L} \leq \mathbf{A} \mathbf{w} \leq \mathbf{a}_{U}
$$

for some matrix $\mathbf{A}$ and vectors $\left(\mathbf{a}_{L}, \mathbf{a}_{U}\right)$. Obviously, this will enforce diversification through portfolios that do not take extreme positions and improve out-of-sample performance as Frost and Savarino (1988) show by means of simulations. More recently, Jagannathan and Ma (2003) point out that short-sale constraints might decrease portfolio risk even when they are wrong (they are not satisfied by the corresponding true portfolio).

The use of constraints is not very satisfactory a priori. They are imposed because of bad quality of the inputs and hence improving the quality of the inputs should be a more fruitful avenue. However, Jagannathan and Ma (2003) show that certain constraints are equivalent to a modified estimation of $\boldsymbol{\Sigma}$ that can be interpreted as shrinkage estimation. This method of estimation is based on a convex combination of a sample estimator and some constrained estimator, and hence the sample estimator is "shrinked" to the constrained one. This estimation tries to find an optimal trade-off between the induced bias and the gain in sampling variance.

Applications of shrinkage estimation to portfolio choice can be found in Jorion (1986), who computes an optimal shrinkage estimator of the mean that combines the sample mean and a common mean, and Ledoit and Wolf (2003), who apply shrinkage estimation to the variance, computing the optimal weight between the sample variance and the estimator derived from a single-factor model. Jorion (1986) motivates the use of shrinkage estimation in the context of a Bayesian investor, where there is a natural interpretation of such method, and this will be studied in Section 4.3.

Most part of the references of this section are closer to security selection than asset allocation since they study the case of a high $N$, e.g. construction of a portfolio of many stocks. In that

\footnotetext{
${ }^{4}$ This result should be carefully applied. Aït-Sahalia et al. (2005) show that microstructure noise implies an optimal finite frequency, unless that noise is also modelled and estimated. Then the highest frequency is optimal again.
} 
context, there are many unknown parameters and the estimation error has stronger implications. Factor models are the usual choice to handle the high number of parameters in $\boldsymbol{\Sigma}$. Chan et al. (1999) evaluate factor models of $\boldsymbol{\Sigma}$ in terms of out-of-sample performance of optimal portfolios. The reader can find additional information on shrinkage methods, factor models, and portfolio constraints in Section 3.1.2 of Brandt (2004).

\subsection{Sampling Uncertainty in Portfolios}

We have followed classic inference in our approximation to our lack of knowledge of true mean-variance parameters. Let us make explicit what we have done so far in the context of a single risky asset. We have a time series of its excess returns $\left(e_{1}, e_{2}, \ldots, e_{T}\right)$, which we represent by $e^{T}$. We assume a simple normal model with independent and identically distributed (i.i.d.) observations, i.e. conditional on some value of the parameters $\left(\mu, \sigma^{2}\right)$

$$
e_{t} \mid\left(\mu, \sigma^{2}\right) \sim N\left(\mu, \sigma^{2}\right), \quad t=1,2, \ldots, T
$$

We leave time dependence in returns for Section 6. Since we commented that an accurate estimation of the variance is easier, we assume we know $\sigma^{2}$ to simplify and hence our analysis will be conditional on its value. We are interested in the distribution of the next-period excess return given our information to compute the optimal portfolio. In the previous model, this distribution would be

$$
e_{T+1} \mid\left(\mu, \sigma^{2}, e^{T}\right) \sim N\left(\mu, \sigma^{2}\right)
$$

if we knew $\mu$. Unfortunately, we do not know $\mu$ and the traditional approach applies a plug-in estimation in the sense of using the distribution $N\left(\hat{\mu}, \sigma^{2}\right)$ with some estimator $\hat{\mu}$. This is a mean-variance framework and the optimal portfolio is given by (5). The estimator $\hat{\mu}$ can be the maximum likelihood one, i.e. the statistic that maximizes $\ln p\left(e^{T} \mid \mu, \sigma^{2}\right)$ with respect to $\mu$, where $p(\cdot)$ is the likelihood. In the previous normal model, the maximum likelihood estimator is simply the sample mean $\hat{\mu}=\bar{e}=T^{-1} \sum_{t=1}^{T} e_{t}$.

If we base the computation of an optimal portfolio on sample data as commented above then the portfolio is an statistic with some sampling error. Jobson and Korkie (1980) studied the estimation of mean-variance portfolios' weights, mean, and variance. They approximate their mean and variance and compute their asymptotic distribution under the assumption of i.i.d. normal returns. 
Later, Jobson and Korkie (1983) interpreted the estimation of some mean-variance parameters and mean-variance efficiency tests in an OLS set-up. If we define $\mathbf{E}$ as a $T \times N$ matrix with excess return data and run OLS of a $T \times 1$ vector of ones onto $\mathbf{E}$, we get the estimator

$$
\hat{\mathbf{w}}=\left(\mathbf{E}^{\prime} \mathbf{E}\right)^{-1} \mathbf{E}^{\prime} \mathbf{1}=\left(\frac{1}{1+\hat{\boldsymbol{\mu}}^{\prime} \hat{\boldsymbol{\Sigma}}^{-1} \hat{\boldsymbol{\mu}}}\right) \hat{\boldsymbol{\Sigma}}^{-1} \hat{\boldsymbol{\mu}} .
$$

We only have to rescale the regression coefficients $\hat{\mathbf{w}}$ to get the efficient portfolio (5) estimator

$$
\hat{\mathbf{w}}(\lambda)=\lambda \hat{\boldsymbol{\Sigma}}^{-1} \hat{\boldsymbol{\mu}}
$$

expressed here in terms of risk tolerance $\lambda$. The regression framework gives the corresponding standard errors of $\hat{\mathbf{w}}$ and hence we can easily construct confidence intervals and tests on $\mathbf{w}(\lambda)$, e.g. we can test if some entries are significantly different from zero. More recently, Britten-Jones (1999) works with the OLS set-up too and develops finite-sample inference under i.i.d. normal returns.

The bootstrap (or resampling methods) is an alternative approach to evaluate sampling uncertainty. ${ }^{5}$ This approach can be implemented in a nonparametric or a parametric set-up. The former is based on resampling data with replacement (or more refined approaches derived for time series), while the latter is based on Monte Carlo simulation from a parametric estimate of the return distribution. Jorion (1992) uses the parametric bootstrap implementation assuming i.i.d. normal returns to analyze the sampling variability in optimal portfolios and perform efficiency tests under short-sale constraints.

Michaud (1998) uses bootstrap methods to compute optimal portfolios themselves instead of standard errors, and defines this approach as resampled efficiency. A resampled efficient portfolio associated to some risk tolerance $\lambda$ is an average of mean-variance efficient portfolios (5) associated to that $\lambda$ for $S$ different boostrapped samples with sample moments $\left(\boldsymbol{\mu}_{1}, \boldsymbol{\Sigma}_{1}\right)$, $\left(\boldsymbol{\mu}_{2}, \boldsymbol{\Sigma}_{2}\right), \ldots,\left(\boldsymbol{\mu}_{S}, \boldsymbol{\Sigma}_{S}\right)$

$$
\begin{gathered}
\mathbf{w}_{r}(\lambda)=\frac{1}{S} \sum_{s=1}^{S} \mathbf{w}_{s}(\lambda), \\
\mathbf{w}_{s}(\lambda)=\lambda \boldsymbol{\Sigma}_{s}^{-1} \boldsymbol{\mu}_{s}, \quad s=1,2, \ldots, S .
\end{gathered}
$$

The size of boostrapped samples can be different from the original data to reflect high or low confidence on historical estimates. Resampled portfolios show higher diversification and less

\footnotetext{
${ }^{5}$ See Horowitz (2001) for a survey on bootstrap methods.
} 
sharp changes with respect to $\lambda$ than traditional mean-variance portfolios, which translates into a better out-of-sample performance. However, this is a heuristic method to deal with estimation error without a theoretical justification as Scherer (2002) points out.

\subsection{Bayesian Portfolio Choice}

This section will be devoted to a Bayesian treatment of parameter uncertainty instead of the previous classic inference. Bayesian inference is a formal way of dealing with estimation error in problem (3) with a strong foundation in decision theory, where we study beliefs and learning after observing a sample instead of working with estimators and their sampling distribution. In fact, Markowitz (1952) does not impose that the mean-variance inputs are the true ones and simply works with beliefs.

A different approach with axiomatic foundations is robust choice. A robust investor follows a maximin rule, choosing the best portfolio in the worst scenario. See Garlappi et al. (2007) and Lutgens and Schotman (2007) for recent references related to mean-variance analysis.

We return to the simple model at the beginning of Section 4.2, where there is a single normal risky asset and we know $\sigma^{2}$. This is one of the simplest examples of Bayesian mechanics and does not represent the power of the approach. On the other hand, it will help to motivate the shrinkage estimation commented in Section 4.1 and the Black-Litterman formulas that are shown later in this section. Bayesian inference needs two inputs and produces two outputs:

- Inputs: One input is the data likelihood $p\left(e^{T} \mid \mu, \sigma^{2}\right)$, i.i.d. normal returns in our example, which represents sample information and is common to classic inference. The second input is the prior beliefs $p\left(\mu, \sigma^{2}\right)$, our beliefs about $\left(\mu, \sigma^{2}\right)$ before observing $e^{T}$. In our simple example, we know $\sigma^{2}$ and we will use the following prior $p(\mu)$

$$
\mu \sim N\left(\rho_{0}, \varphi_{0}\right)
$$

where the prior mean $\rho_{0}$ could be derived through a theoretical model such as the CAPM, and the prior variance $\varphi_{0}$ would measure our confidence in that model.

- Outputs: The first output is the posterior beliefs after observing $e^{T}, p\left(\mu, \sigma^{2} \mid e^{T}\right)$, which follows Bayes' rule to combine prior beliefs and sample information given by the data 
likelihood. ${ }^{6}$ In our example, we are interested in $p\left(\mu \mid \sigma^{2}, e^{T}\right)$

$$
\begin{gathered}
\mu \mid\left(\sigma^{2}, e^{T}\right) \sim N\left(\rho_{T}, \varphi_{T}\right), \\
\varphi_{T}=\left(\frac{1}{\varphi_{0}}+\frac{T}{\sigma^{2}}\right)^{-1}, \quad \rho_{T}=\varphi_{T}\left(\frac{\rho_{0}}{\varphi_{0}}+\frac{T \bar{e}}{\sigma^{2}}\right),
\end{gathered}
$$

where the expression of $\rho_{T}$ can be interpreted as shrinking $\bar{e}$ towards $\rho_{0}$. On the other hand, these posterior beliefs are not our main object of interest. The portfolio choice problem in (3) requires the distribution of next-period returns given $e^{T}, p\left(e_{T+1} \mid e^{T}\right)$, which is the second output and is called the predictive distribution. ${ }^{7}$ The previous i.i.d. normal model states that $e_{T+1} \mid\left(\mu, \sigma^{2}, e^{T}\right) \sim N\left(\mu, \sigma^{2}\right)$ and the relevant predictive density $p\left(e_{T+1} \mid \sigma^{2}, e^{T}\right)$ integrates out our uncertainty about $\mu$. In this simple model, the predictive is still normal and hence the solution to $(3)^{8}$ is the mean-variance solution (5) for the particular mean and variance in

$$
e_{T+1} \mid\left(\sigma^{2}, e^{T}\right) \sim N\left(\rho_{T}, \sigma^{2}+\varphi_{T}\right)
$$

Barry (1974) is one the first references that study the effect of estimating the mean and/or the variance in the predictive distributions of a portfolio choice problem. In those models, we are still in a mean-variance framework and the variance matrix is simply scaled. Therefore, estimation risk changes the selected portfolio but does not change the efficient set. Klein and Bawa (1976) work with non-informative priors, which give a Student-t predictive distribution with similar implications to Barry (1974), but also consider informative priors in special cases that change the efficient set. Later, Frost and Savarino (1986) use a Bayesian set-up in the spirit of shrinkage estimators by means of a prior where all means, variances and correlations are equal.

Pástor (2000) and Pástor and Stambaugh (2000) represent more recent references. Their priors are based on factor pricing models and their likelihood is also given by i.i.d. normal returns.

\footnotetext{
${ }^{6}$ The general expression when we do not know $\sigma^{2}$ is

$$
p\left(\mu, \sigma^{2} \mid e^{T}\right) \propto p\left(\mu, \sigma^{2}\right) p\left(e^{T} \mid \mu, \sigma^{2}\right),
$$

where $\propto$ means proportional to.

${ }^{7}$ The general expression when $\sigma^{2}$ is unknown integrates out $\left(\mu, \sigma^{2}\right)$

$$
p\left(e_{T+1} \mid e^{T}\right)=\int_{\mathbb{R}_{+}} \int_{\mathbb{R}} p\left(e_{T+1} \mid \mu, \sigma^{2}, e^{T}\right) p\left(\mu, \sigma^{2} \mid e^{T}\right) \mathrm{d} \mu \mathrm{d} \sigma^{2} .
$$

${ }^{8}$ Some combinations of vN-M utility and Bayesian inference might not give a well defined expected utility. See Geweke (2001) for some examples on CRRA utility.
} 
Pástor (2000) evaluates home bias and value and size effects by means of asset allocation. Pástor and Stambaugh (2000) compare asset pricing models by means of their asset allocation implications when they are used as priors. They also comment briefly the case of model uncertainty in addition to parameter uncertainty because it can also be incorporated by means of Bayesian model averaging. Tu and Zhou (2004) extend the Pástor and Stambaugh (2000) framework to returns that follow a Student-t and there is uncertainty about the degrees of freedom.

We have only commented computations and references related to portfolio construction, but not to the corresponding equilibrium implications. Lintner (1969) represents one of the first approximations to equilibrium under heterogenous expectations in a mean-variance set-up. He shows that the CAPM holds with "market representative" beliefs even though that agents hold different portfolios. Barry and Brown (1985) study the beta implications of a Bayesian set-up where there is more information available for some assets than others and also different information across agents. However, beliefs are exogenous in those references and hence they do not define a rational expectations equilibrium. The book Brunnermeier (2001) is a good introduction to the complex issue of asset pricing under asymmetric information.

The rest of this section will be devoted to the Black-Litterman approach, which is similar in spirit to the Bayesian approach and is widely used in the industry nowadays. Black and Litterman (1992) explicitly assume that the historical variance is a good estimator but not the sample mean. They estimate the risk premia by means of a combination of equilibrium and individual views, in such a way that the market portfolio acts as a well-behaved anchor.

Our exposition will mainly follow He and Litterman (1999). The reader can find a revision of several issues of investment management under this approach in Litterman (2003) and the introduction of additional views on volatilities and correlations in a slightly different set-up in Qian and Gorman (2001). Let us return to our general situation of $N$ risky assets:

- Inputs: An equilibrium model and investor's views. The first input defines beliefs on risk premia as

$$
\boldsymbol{\mu} \sim N(\boldsymbol{\pi}, \tau \hat{\boldsymbol{\Sigma}})
$$

where $\hat{\boldsymbol{\Sigma}}$ is some estimated variance matrix of excess returns using historical data, $\tau$ reflects the degree of (lack of) confidence on equilibrium returns (we might choose it as $T^{-1}$, where $T$ is interpreted as a sample size), and $\boldsymbol{\pi}$ are the implied risk premia from the market portfolio under the CAPM. For some plausible risk tolerance $\lambda$, we can invert the 
mean-variance portfolio choice (5) evaluated at the market portfolio $\mathbf{w}_{M}$

$$
\boldsymbol{\pi}=\left(\frac{1}{\lambda}\right) \hat{\mathbf{\Sigma}}_{\mathbf{w}_{M}}
$$

which is and equivalent expression to the CAPM equation (8) if we note that $\lambda=\sigma_{M}^{2} / \mu_{M}$. The second piece of information is expressed as some subjective views on risk premia. The investor might have several views on linear combinations of risk premia that we can express by means of

$$
\mathbf{Q} \boldsymbol{\mu} \sim N(\mathbf{q}, \mathbf{\Omega})
$$

where $\boldsymbol{\Omega}$ reflects the degree of confidence on those views. This matrix is usually chosen to be diagonal and its diagonal entries can be composed by scaled historical variances as we commented above for $\tau \hat{\boldsymbol{\Sigma}}$.

- Outputs: The equilibrium information might be interpreted as the prior beliefs and the subjective views as additional information. Both pieces of information are combined into a posterior-like distribution on risk premia

$$
\begin{gathered}
\boldsymbol{\mu} \sim N\left(\boldsymbol{\pi}_{c}, \boldsymbol{\Sigma}_{c}\right), \\
\boldsymbol{\Sigma}_{c}=\left[\mathbf{Q}^{\prime} \mathbf{\Omega}^{-1} \mathbf{Q}+(\tau \hat{\boldsymbol{\Sigma}})^{-1}\right]^{-1}, \quad \boldsymbol{\pi}_{c}=\boldsymbol{\Sigma}_{c}\left[\mathbf{Q}^{\prime} \mathbf{\Omega}^{-1} \mathbf{q}+(\tau \hat{\boldsymbol{\Sigma}})^{-1} \boldsymbol{\pi}\right],
\end{gathered}
$$

which is similar to (11) in a general Bayesian framework. The corresponding predictive-like distribution is normal and similar to $(12)$

$$
e_{T+1} \sim N\left(\boldsymbol{\pi}_{c}, \hat{\boldsymbol{\Sigma}}+\boldsymbol{\Sigma}_{c}\right)
$$

The portfolio advocated by this approach applies (5) to that distribution

$$
\mathbf{w}_{c}(\lambda)=\lambda\left(\hat{\boldsymbol{\Sigma}}+\boldsymbol{\Sigma}_{c}\right)^{-1} \boldsymbol{\pi}_{c}
$$

and the computed portfolios are not as extreme as in traditional mean-variance. If there is a low confidence in subjective views with respect to equilibrium (or simply neutral views) then the investor holds the market portfolio. Specifically, as $\boldsymbol{\Omega}$ grows without bound there is more weight in equilibrium vs. views and $\mathbf{w}_{c}(\lambda)$ converges to $\lambda(\hat{\boldsymbol{\Sigma}}+\boldsymbol{\tau} \hat{\boldsymbol{\Sigma}})^{-1} \boldsymbol{\pi}=(1+\tau)^{-1} \mathbf{w}_{M}$. 


\section{Beyond Mean-Variance Preferences}

\subsection{From Expected Utility to Moments of Returns}

Our general approach to portfolio choice has been the expected utility paradigm, represented by (3). Now we are going to study the connection between expected utility and the properties of returns distributions. We can express a general vN-M utility function $u(\cdot)$ through its Taylor expansion around the mean of the portfolio return $R$ and take expectations

$$
\begin{gathered}
E[u(R)]=u(1+v)+\left(\frac{1}{2}\right) \frac{\mathrm{d}^{2} u(1+v)}{\mathrm{d} r^{2}} E\left[(r-v)^{2}\right] \\
+\left(\frac{1}{3 !}\right) \frac{\mathrm{d}^{3} u(1+v)}{\mathrm{d} r^{3}} E\left[(r-v)^{3}\right]+\left(\frac{1}{4 !}\right) \frac{\mathrm{d}^{4} u(1+v)}{\mathrm{d} r^{4}} E\left[(r-v)^{4}\right]+\ldots .
\end{gathered}
$$

assuming the utility function is smooth enough and the moments finite. Therefore, expected utility depends on the mean, variance, skewness, kurtosis, etc. of portfolio returns.

There are two cases where we can rely on mean and variance and skip the rest of moments, i.e. $E[u(R)]=U\left(\nu, \sigma^{2}\right)$. In terms of $\mathrm{vN}-\mathrm{M}$ utility $u(\cdot)$, if this function is quadratic then the investor only cares about mean and variance. But this utility is not a plausible description of investor behaviour since it shows decreasing marginal utility from some point and increasing absolute risk aversion (a risky asset is an inferior good).

The second case relies on the distribution of returns. If returns are multivariate normal and hence final wealth is normal, $R \sim N\left(1+\nu, \sigma^{2}\right)$, then mean and variance fully describe the relevant distribution. In fact, mean-variance analysis is not only constrained to normal returns. Risky assets following a multivariate elliptical distribution is a more general justification as Chamberlain (1983) showed. He also showed that if a riskless asset is not available then risky assets can have a more general distribution. Owen and Rabinovitch (1983) studied the link between elliptical returns and mean-variance analysis too.

The fact that a Student-t distribution is also compatible with mean-variance analysis (assuming the variance exists) points out that it is skewness or asymmetry, but not kurtosis or fat tails the actual problem for the use of variance as an accurate measure of risk. Skewness is problematic when we work with options or under limited liability at a theoretical level. In addition, there is a considerable amount of empirical evidence on asymmetric returns documented by the references of this section. ${ }^{9}$ However, Peiró (1999) questions such evidence with daily returns of some stock markets and currencies. The usual empirical evidence against symmetry is based

\footnotetext{
${ }^{9}$ This refers to the plausibility of normal univariate returns, but there is a multivariate dimension of asymmetry
} 
on sample skewness, but this test might not be quite reliable under non-normal distributions and he does not find clear evidence against symmetry when using other types of tests. On the other hand, those tests are based on i.i.d. log-returns. Sánchez-Torres and Sentana (1998) use a skewness test that does not rely on i.i.d. normal returns. More recently, Bai and Ng (2005) propose similar moment tests of skewness and kurtosis with time series data that are easy to apply.

Nevertheless, we can still rely on mean-variance analysis as an approximation to choice under expected utility. We can interpret mean-variance preferences $U\left(\nu, \sigma^{2}\right)$ as the second order approximation

$$
E[u(R)] \simeq u(1+v)+\left(\frac{1}{2}\right) \frac{\mathrm{d}^{2} u(1+v)}{\mathrm{d} r^{2}} \sigma^{2}
$$

and its ranking accuracy will depend on the vN-M utility function and return distribution. This is an empirical question that will depend on the particular situation under study, e.g. the asset classes and the horizon that is considered. We will focus on the former example since the role of the investment horizon will be analyzed in Section 6.1.

Levy and Markowitz (1979) find that the approximation performs well in terms of ranking mutual funds. Kroll et al. (1984) extend the analysis to portfolio choice with stocks and compare the corresponding optimal portfolio, showing that mean-variance analysis works well with nonnormal returns. Hlawitschka (1994) also extends Levy and Markowitz (1979) in an application to derivatives, which are clearly non-normal. He shows a high rank correlation between meanvariance and other preferences and points out that third and fourth-order Taylor expansions do not necessarily improve the portfolio choice.

There are asset classes such as of emerging markets' stocks and bonds and more recently hedge funds that might be quite attractive in mean-variance terms due to low correlations with other asset classes and high Sharpe ratios. But this analysis forgets that they might have a significant negative asymmetry (and high kurtosis) and the investor might dislike it. Bekaert et al. (1998) study asset allocation with cash, stocks and emerging markets when the investor has power utility. On the other hand, Fung and Hsieh (1999) show an application to hedge-funds where mean-variance analysis preserves approximately the ranking of other preferences.

Harvey et al. (2004) and Jondeau and Rockinger (2006) are two recent references of portfolio that is not always made explicit. For instance, Ang and Chen (2002) find asymmetric correlation in equity portfolios, in the sense of a higher correlation between individual US stocks and the aggregate US market for downside moves than for upside moves. 
choice without mean-variance preferences. Harvey et al. (2004) analyze the Bayesian portfolio choice of Section 4.3 in this new set-up. They use a skew normal distribution to model returns, which gives both asymmetry and fat tails. Jondeau and Rockinger (2006) use Taylor expansions of expected (exponential) utility up to fourth order to easily handle asset allocation that takes into account skewness and kurtosis. Mean-variance portfolios are close to the optimal ones with weekly data on stock indices of different regions. However, they are a bad approximation with weekly data on individual stocks unless risk aversion is low, while introducing skewness and kurtosis alleviates the problem.

Finally, let us comment the equilibrium implications of a framework that does not rely on mean-variance preferences. Ingersoll (1987) dedicates Chapter 6 to mutual-fund separation results under restrictions on vN-M utilities or return distributions, and their respective pricing implications. That chapter gives the relevant references for a series of important issues: Twofund separation holds under more general set-ups than quadratic vN-M utility and/or elliptical returns and there are non-elliptical distributions such that the CAPM holds; on the other hand, some of the results that look natural in a mean-variance set-up are no longer true for general preferences, e.g. the efficient portfolio set is not necessarily convex and hence the market portfolio might be inefficient.

Rubinstein (1973) is one of the first references that extend the CAPM to preferences that take into account additional moments. Krawss and Litzenberger (1976) start from preferences that depend on the first three moments of returns and develop a pricing equation similar to (8) that requires a new term of coskewness with the market. Simaan (1993) introduces skewness in a factor model of returns. There is three-fund separation in his model, where the third fund is related to skewness risk, and this translates into an additional term in the pricing equation (8). More recently, Harvey and Siddique (2000) take into account coskewness with the market as an additional pricing factor.

\subsection{Lower Partial Moments}

The previous section studied the connection between mean-variance preferences and expected utility, stressing that skewness is missing in mean-variance analysis (already mentioned by Markowitz (1952)). If two assets with the same mean and variance have different skewness then we expect that an investor prefers the one with higher (positive) skewness. 
There have been several approaches that introduce preference for positive skewness in a set-up as close as possible to mean-variance analysis. For instance, Ingersoll (1975) introduces skewness as a third dimension in the analysis and recently De Athayde and Flôres (2004) characterize the efficient set in such a context. This section will focus in a different approach that also follows the mean-variance spirit.

We can consider variance as an overall or absolute risk measure, in the sense that it takes into account both positive and negative outcomes. Our main concern is this might not be an accurate risk measure in general and hence another approach is the use of another risk measure in the problem (4).Now we will work with downside risk measures instead, i.e. we will understand risk as falling below a reference point or shortfall risk. Specifically, we can use a lower partial moment (LPM) for a reference point $r_{*}$ and order $k$

$$
L P M_{k}\left(r_{*}\right)=E\left[\left[\operatorname{Max}\left(r_{*}-r, 0\right)\right]^{k}\right]=E\left[\left(r_{*}-r\right)^{k} I\left(r \leq r_{*}\right)\right],
$$

where $I(A)$ is the indicator function that returns 1 if $A$ is true and 0 otherwise. The reference point $r_{*}$ can be the risk-free rate, a benchmark, inflation, etc.

The choice of $k=0$ gives $L P M_{0}\left(r_{*}\right)=\operatorname{Pr}\left(r \leq r_{*}\right)$, the shortfall probability, which was already proposed by Roy (1952) as the safety first approach. A related but different approach is to choose value at risk $(\mathrm{VaR})$ as the risk measure. $\mathrm{VaR}$ is the quantile given by a lefttail probability instead and is the most famous downside risk measure in risk management. Obviously, now we are getting close to risk management since we are looking for an accurate risk measure. However, expected utility is always our motivation because we are studying portfolio choice. Alexander and Baptista (2002) study portfolio choice under mean-VaR preferences, characterizing the corresponding frontier and the equilibrium implications.

Bawa (1978) extended the approach in Roy (1952) to a general nth-order LPM and studied the relationship of such a decision rule with stochastic dominance. Chapter 2 of Huang and Litzenberger (1988) describes stochastic dominance, a criterion to discriminate portfolios under the expected utility paradigm that is only based on the signs of the derivatives of an otherwise general vN-M utility function $u(\cdot)$ (say a risk averse investor). Obviously, this criterion is much more general than mean-variance analysis. Unfortunately, it is not a practical rule, while LPMs represent a computable approximation. Bawa considers LPMs as a better risk measure than variance for that reason. More recently, Daníelsson et al. (2006) revise the connection between stochastic dominance and several (overall and downside) risk measures. 
An obvious critique to $k=0$ is that it does not take into account the level of underperformance. However, $k=1$ does since $L P M_{1}\left(r_{*}\right)=\operatorname{Pr}\left(r \leq r_{*}\right) E\left[r_{*}-r \mid r \leq r_{*}\right]$ and the underperformance average shows up. The conditional mean term in $L P M_{1}\left(r_{*}\right)$ is related to concepts such as expected shortfall, conditional VaR, tail conditional expectation, or expected tail loss in risk management. The reader can find their precise definition in Daníelsson et al. (2006).

We can also criticize the choice of $k=1$ since it shows risk neutrality under $r_{*}$. MeanLPM preferences can be represented with expected utility by means of a vN-M utility $u(R)=$ $R-\theta\left(r_{*}-r\right)^{k} I\left(r \leq r_{*}\right)$ for some $\theta>0$, with a corresponding expected utility $E[u(R)]=$ $(1+\nu)-\theta L P M_{k}\left(r_{*}\right)$. A general order $k$ is linked to risk aversion in the sense that $k=1$ separates risk-seeking from risk-averse behaviour for $r \leq r_{*}$. If we move to $k=2$ then we find the shortfall semivariance $L P M_{2}\left(r_{*}\right)$, already commented by Markowitz (1959) as a more robust risk measure. The concept of semivariance is sometimes reserved for $r_{*}=E(r)$. Under a symmetric distribution, $\operatorname{LPM}_{2}(E(r))=0.5 \sigma^{2}$ and hence the ordering would be the same using mean-variance preferences.

Let us focus on $r_{*}=r_{0}$ and the semivariance $L P M_{2}\left(r_{0}\right)$. Then we can express the optimal portfolio in a mean-LPM framework as the solution to

$$
\min _{\mathbf{w}} L P M_{2}\left(r_{0}\right) \quad \text { s.t. } \quad E(e)=\mu \text {. }
$$

Hogan and Warren (1972) develop some theoretical results about the mean-semivariance frontier without a safe asset and a general $r_{*}$. They show that $L P M_{2}\left(r_{*}\right)$ is convex and continuos differentiable in w. Therefore, this is a standard optimization problem even though we do not have a explicit solution like in the mean-variance context (recall (5)). The frontier is convex on $\left(\nu, L P M_{2}\left(r_{*}\right)\right)$ space. Later, Hogan and Warren (1974) study the existence of a riskless asset and $r_{*}=r_{0}$. The concepts of two-fund spanning and tangency portfolio apply also in this context and the efficient frontier is a straight line on space $\left(\mu, L P M_{2}^{1 / 2}\left(r_{0}\right)\right)$, i.e. we find a similar situation to Figure 2 .

Bawa and Lindenberg (1977) comment similar results for the order $k=1$ too when $r_{*}=r_{0}$, while Harlow and Rao (1989) analyzed a general $r_{*}$. Harlow (1991) reviews the mean-LPM analysis and constructs an empirical application to international asset allocation. There is a higher investment in bonds than with mean-variance preferences. Sortino and Forsey (1996) advocate the use of semivariance but comment some problems with its implementation. 
Finally, let us describe the equilibrium implications of mean-LPM preferences. Hogan and Warren (1974) study the translation of the CML and SML to the mean-semivariance context when $r_{*}=r_{0}$. As expected from previous comments about the mean-semivariance frontier, there is two-fund separation using the safe asset and the market portfolio, and the main novelty is that the pricing equation (8) should use a different beta. Its numerator should be the cosemivariance with the market, where the truncation is $e_{M} \leq 0$ (the cosemivariance is not a symmetric concept, while the covariance is), and its denominator should be the market semivariance.

Bawa and Lindenberg (1977) comment similar results for the order $k=1$ too when $r_{*}=$ $r_{0}$, while Harlow and Rao (1989) analyzed a general $\tilde{r}$. They also develop an econometric implementation of the model and their empirical results show that this model performs better than the traditional CAPM. More recently, Pedersen and Satchell (2002) review the equilibrium implications of mean-LPM preferences as a generalization of quadratic vN-M utility instead of elliptical distributions. The CAPM holds under two-fund separating distributions, which are more general than elliptical distributions, and the mean-LPM pricing does not add value in those situations.

\subsection{Alternative Performance Measures to Sharpe Ratio}

We will dedicate our last comments on developments beyond mean-variance preferences to some alternatives to the Sharpe ratio, defined in (7). The main goal of those measures is to give a similar ranking to Sharpe ratios when returns are symmetrically distributed and show a preference for skewness when they are not. Moreover, performance measures can be used to guide asset allocation since they can be used as the criterion to maximize with portfolio.

A natural generalization of the Sharpe ratio of a risky security is the Sortino ratio

$$
S\left(r_{*}\right)=\frac{v-r_{*}}{L P M_{2}^{1 / 2}\left(r_{*}\right)},
$$

which is based on semivariance for some threshold $r_{*}$ as the risk measure. Pedersen and Satchell (2002) study the theoretical foundations of this measure and advocate the use of $r_{*}=r_{0}$. In that context, we commented that the efficient frontier satisfies the properties of two fund spanning and linearity on $\left(\mu, L P M_{2}^{1 / 2}\left(r_{0}\right)\right)$. This linearity provides a single optimal trade-off between risk and return that can discriminate portfolio efficiency, as it is the case with the Sharpe ratio.

A different performance measure based on the ratio of upper to lower partial moments of order $k=1$ is proposed in Keating and Shadwick (2002), but it is a function of the threshold $r_{*}$ 
and it lacks a theoretical justification. They define the Omega function by

$$
\Omega\left(r_{*}\right)=\frac{E\left[\operatorname{Max}\left(r-r_{*}, 0\right)\right]}{E\left[\operatorname{Max}\left(r_{*}-r, 0\right)\right]}=\frac{U P M_{1}\left(r_{*}\right)}{L P M_{1}\left(r_{*}\right)}
$$

and study its properties. Its ranking of assets with different variance, skewness, or kurtosis depends on $r_{*}$, e.g. omega might increase with variance when $r_{*}$ is high.

Stutzer (2000) develops a performance measure that is based on the hypothesis that a fund manager is averse to receiving a nonpositive time-averaged excess return above some benchmark. Specifically, her objective is to minimize the probability of underperformance over the next $T$ periods, which is $\operatorname{Pr}(\bar{e} \leq 0)$ is we simply assume that the benchmark is the risk-free asset. This criterion is close to Roy's safety-first approach.

If portfolio returns are i.i.d (this can be generalized) and satisfy $\mu>0$ then a law of large numbers shows that $\operatorname{Pr}(\bar{e} \leq 0) \simeq 0$ for a high horizon $T$. This fact is not very useful to order portfolios and a central limit theory does not provide a good approximation to that probability, but we can rely on large deviation theory instead. This theory gives the rate of decay of that probability

$$
\operatorname{Pr}(\bar{e} \leq 0) \simeq \exp (-I \cdot T), \quad I=\max _{\theta}-\ln E[\exp (\theta e)],
$$

which is related to the moment generating function of $e$, assuming it is well defined.

The rate of decay $I \geq 0$ ( $I>0$ if $\mu>0$, and $I=0$ otherwise) is the proposed performance index, which gives the same ranking as the Sharpe ratio under normality because $I=0.5 S R^{2}$ in that case. Stutzer faces the portfolio choice with criterion $I$ against a Sharpe ratio criterion with several stocks and finds that the portfolio chosen by $I$ shows some skewness preference that is not taken into account by the Sharpe ratio.

The usefulness of these alternatives is an empirical question. Recently, Eling and Schuhmacher (2007) face the Sharpe ratio ranking of hedge funds against twelve alternative measures that include Omega and the Sortino ratio for instance. Hedge funds are clearly non-normal, but the Sharpe ratio gives a ranking that is similar to the other measures.

\section{Multi-Period Choice}

\subsection{Long-Term Choice and Predictability}

We have assumed a particular horizon in the previous sections but we have not been explicit about it. Now we will study the effect of the horizon, which will be denoted by $T$ periods, 
in buy-and-hold strategies. A textbook treatment of the following concepts can be found in Chapter 2 of Campbell and Viceira (2002).

First, we note that there is not a universal long-term portfolio that is optimal for every agent even though the growth-optimal portfolio might look so. That portfolio maximizes the expected geometric return $E\left[T^{-1} \ln \left(R_{1} R_{2} \ldots R_{T}\right)\right]$ and hence $E\left[T^{-1} \ln \left(W_{T}^{g} / W_{T}\right)\right]>0$, where $W_{T}^{g}$ is the final wealth from the growth-optimal portfolio and $W_{T}$ is the final wealth of any other portfolio. If we assume i.i.d. returns and apply the corresponding law of large numbers then the growth-optimal portfolio outperforms any other portfolio in the long run (as $T$ goes to infinity) with probability reaching one.

However, this choice does not necessarily maximize expected utility for preferences different to vN-M $\log$-utility $u(R)=\ln R$. Similarly, we can also translate this argument to a meanvariance context because $E(\ln R) \simeq(1+\nu)-0.5 \sigma^{2}$ and hence its maximization is optimal for a risk tolerance of 1 , which represents a fairly aggressive investor. Stutzer (2003) is a related reference where the application of large deviation theory in Stutzer (2000) is linked to longhorizon portfolio choice.

Another natural question is the behaviour of the mean-variance frontier as investment horizon lengthens. ${ }^{10}$ The answer is that it does not change with the number of periods $T$ when returns are i.i.d. To simplify, let us assume a single risky asset class, say the stock market, and that long-term returns are approximately equal to the sum of short-term returns. Then the efficient portfolio (5) for horizon $T$ expressed in terms of risk tolerance $\lambda$ is

$$
w(\lambda) \simeq \lambda \frac{\mu T}{\sigma^{2} T}=\lambda \frac{\mu}{\sigma^{2}}
$$

that is, it is independent of horizon since both risk premium and variance grow linearly with time in this random walk context. Obviously, the previous expression is an approximation and there might be some horizon effects, but they are not relevant in practice. The previous expression is exact in continuous time with diffusions.

This horizon-independence points out a potentially misleading use of Sharpe ratios. The

\footnotetext{
${ }^{10}$ Another interesting but slightly different question on horizon effects is the shape of the distribution of returns, which is related to Section 5.1. Arditti and Levy (1976) studied skewness in multi-period returns under i.i.d. returns. More recently, Levy and Duchin (2004) use goodness-of-fit tests of several models at different horizons for both stocks and bonds. The best fitting distributions change from short to long horizons: Elliptical distributions fit well at short horizons and hence mean-variance analysis would be justified, but some positive skewness is found at long horizons.
} 
Sharpe ratio of the previous portfolio is $S R \simeq \sqrt{T} \mu / \sigma$ when returns are uncorrelated, that is, it increases with time. However, we should compare Sharpe ratios across assets for a given horizon and we should not compare Sharpe ratios across different horizons. On the other hand, Hodges et al. (1997) show horizon effects in Sharpe ratios of stocks and bonds that signals non-i.i.d. returns. They find that they first increase and lately decrease for each asset and also that the ranking changes.

There are some empirical examples of clear time series dependence in financial returns: Interest rates are autocorrelated and stock returns are conditionally heteroskedastic. We will not discuss the empirical evidence on predictability and market efficiency in this paper. We refer the interested reader to Chapter 20 in Cochrane (2001) and Chapters 2 and 7 in Campbell et al. (1997).

Therefore, we will not think of i.i.d. returns in the rest of the paper and we will take into account (stochastic) time-variation in investment opportunities in portfolio construction. The interest rate $r_{0}$ and the risky assets' risk premia $\boldsymbol{\mu}=E(\mathbf{e})$ and variance matrix $\boldsymbol{\Sigma}=$ $\operatorname{Var}(\mathbf{e})$ should be conditional on the relevant information set (e.g. $E_{t}\left(\mathbf{e}_{t+1}\right)$ and $\operatorname{Var}_{t}\left(\mathbf{e}_{t+1}\right)$, where subscript $t$ denotes conditioning on information up to $t$ ) and not equal to unconditional values. Hansen and Richard (1987) developed a formal framework for mean-variance analysis with conditioning information. That framework can be considered the conditional counterpart of Chamberlain and Rothschild (1983). Ferson and Siegel (2001) and Sentana (2005) represent recent applications of this set-up.

This discussion introduces econometric models of returns time series into portfolio choice. The standard models in financial econometrics can be found in Campbell et al. (1997). Examples of standard models in long-term portfolio choice are $r_{0}$ following an $\operatorname{AR}(1)$ and the risk premium of stocks $\mu$ being linear in the dividend yield. Those examples introduce two of tho most usual predictors (or state variables) in this literature, the short-term interest rate and the dividend yield. Another one is the slope of the term structure of interest rates or simply the term spread.

Campbell and Viceira (2005) use a VAR model of returns and predictors to analyze the variance and correlation of stocks, bonds, and cash (Treasury-bills) across different horizons. The volatility per period decreases with horizon for stocks and increases for cash, which are signals of mean-reversion and reinvestment risk respectively. The global minimum-variance portfolio at different horizons is composed of mostly cash in the short run and mostly stocks 
and bonds in the long run. Guidolin and Timmermann (2006) analyze the term structure of different risk measures such as VaR and expected shortfall under several econometric models such as Markov-switching and GARCH-M with Student-t innovations. There are significant differences across models and there is not a clear best model in an out-of-sample forecasting exercise with portfolios of stocks, bonds, and cash.

The rest of Section 6 will study multi-period asset allocation allowing rebalancing during the investment horizon, i.e. we are interested now in a sequence of portfolio choices instead of a single (buy-and-hold) choice. We will focus on the theoretically optimal strategy developed in the academic literature in Section 6.2 , but we will briefly comment the industry approach before. The reader can find additional details in Sharpe (1987).

The usual practitioner's approach decomposes the portfolio problem in two parts. The first part is called strategic asset allocation (SAA) and defines a long-term target or benchmark. The computation of such portfolio could follow the previous comments on long-term buy-andhold strategies. The second part is called tactical asset allocation (TAA) and tilts the portfolio away from the strategic benchmark to take advantage of short-term changes in investment opportunities. These short-term changes are interpreted as inefficiencies or deviations from equilibrium, and hence an opportunity to active management through market timing, "beating the market" with superior information.

We can apply results from previous sections to this framework. We can use mean-variance analysis to compute the SAA and TAA portfolios, e.g. Clarke and de Silva (1998) show an example with i.i.d. returns and two possible mean-variance regimes. TAA is usually implemented with respect to a benchmark and can follow Section 3.3 and the references therein. The BlackLitterman approach studied in Section 4.3 is also widely used for TAA by the industry, where the market represents the SAA and the investor's views drive the TAA.

\subsection{Dynamic Asset Allocation Theory}

The theoretical optimal strategy is not based on a long-term benchmark and short-term tilts. This approach directly defines a sequence of (contingent) portfolios that is optimal given preferences and return dynamics. We are going to study multi-period portfolio choice in continuous time when returns follow diffusion processes. This context allows to compute more explicit expressions than discrete time and hence helps to clarify the difference to one-period choice. R.C. 
Merton developed the following results in a series of papers that were lately compiled in Merton (2001). Textbook treatments of the following results can be found in Chapter 13 of Ingersoll (1987) (Chapter 11 is dedicated to discrete time portfolio choice) and Chapter 5 of Campbell and Viceira (2002).

Let us assume investment opportunities change over time depending on a random variable $Z$, and therefore it is called state variable or predictor. There are $N$ risky assets that pay no income (only to ease exposition) and their prices are represented by the vector $\mathbf{P}$. Let us denote by $\mathrm{d} \mathbf{Y}$ the vector with entries equal to $\mathrm{d} P / P$ for each asset. At each point in time $t$, prices evolve as

$$
\mathrm{d} \mathbf{Y}=\boldsymbol{\nu}(Z, t) \mathrm{d} t+\boldsymbol{\Gamma}(Z, t) \mathrm{d} \mathbf{B},
$$

where $\mathbf{B}$ is a vector Brownian process and $\boldsymbol{\Gamma}(Z, t)$ is a lower triangular matrix, and the state variable follows a similar equation for $\mathrm{d} Z$ with respect to another scalar Brownian process $B_{Z}$. Drifts (vector $\boldsymbol{\nu}(Z, t)$ for $\mathrm{d} \mathbf{Y}$ ) and volatilities (matrix $\mathbf{\Gamma}(Z, t)$ for $\mathrm{d} \mathbf{Y}$ ) only depend on time and the state variable. ${ }^{11}$ The instantaneous risk-free rate might change with time and the state variable too, $r_{0}(Z, t)$.

Let us define some conditional moments, where some expressions do not make explicit dependence on $(Z, t)$ to simplify notation. The risk premia and the variance matrix are given by

$$
\boldsymbol{\mu} \mathrm{d} t=E_{t}\left(\mathrm{~d} \mathbf{Y}-r_{0} \mathrm{~d} t \mathbf{1}\right)=\left(\boldsymbol{\nu}-r_{0} \mathbf{1}\right) \mathrm{d} t, \quad \boldsymbol{\Sigma} \mathrm{d} t=\operatorname{Var}_{t}(\mathrm{~d} \mathbf{Y})=\boldsymbol{\Gamma} \boldsymbol{\Gamma}^{\prime} \mathrm{d} t
$$

where subscript $t$ denotes conditioning on information up to $t$, and the new relevant object is the (conditional) covariance between prices and the state variable

$$
\gamma \mathrm{d} t=\operatorname{Cov}_{t}(\mathrm{~d} \mathbf{Y}, \mathrm{d} Z)
$$

The portfolio problem is defined as follows. At some initial time 0 , the investor maximizes expected utility of final wealth at some future time $W(T)$ given a budget constraint. She computes a portfolio strategy defined by the function $\mathbf{w}(W, Z, t)$ (continuous rebalancing until

\footnotetext{
${ }^{11}$ A discrete-time counterpart of returns might help to clarify the stochastic structure. We can think of a return process

$$
r_{t+1}=v\left(z_{t}\right)+\sigma\left(z_{t}\right) u_{t+1}
$$

where $v(\cdot)$ and $\sigma(\cdot)$ are functions of the state variable $z_{t}$ (and independent of time to simplify), and $u_{t+1}$ is a standard normal shock. Conditional on $z_{t}, r_{t+1}$ is normal with mean $v\left(z_{t}\right)$ and variance $\sigma^{2}\left(z_{t}\right)$. Finally, we can also think that $z_{t}$ follows an $\operatorname{AR}(1)$ process.
} 
time $T$ ), measured as portfolio weights relative to wealth, that solves the problem

$$
\max _{\mathbf{w}} E_{0}[u(W(T))] \quad \text { s.t. } \quad \mathrm{d} W=W\left[\left(r_{0}+\mathbf{w}^{\prime} \boldsymbol{\mu}\right) \mathrm{d} t+\mathbf{w}^{\prime} \mathbf{\Gamma} \mathrm{d} \mathbf{B}\right]
$$

for some initial wealth $W(0)>0$. Wealth is also constrained to be positive every time. We could introduce consumption (and think of an infinite horizon) but it is not a key issue for our main points.

This problem was solved by Merton (1969) for the case of lack of predictability. They reader can find in Samuelson (1969) its discrete time counterpart. Mossin (1968) also studied the discrete time case by means of dynamic programming. Merton (1971) made several extensions of Merton (1969) and one of them was the introduction of $Z$. He used dynamic programming to solve the problem and that is what we will do. Cox and Huang (1989) show an alternative technique based on martingales to solve the problem that is sometimes easier to apply but requires the assumption of complete markets.

Defining the value function as expected utility at the optimum

$$
V(W, Z, t)=\max _{\mathbf{w}} E_{t}[u(W(T))]
$$

and denoting partial derivatives of $V$ by subindices, the optimal portfolio is implicitly given by ${ }^{12}$

$$
\begin{gathered}
\mathbf{w}_{d}=\lambda_{m} \boldsymbol{\Sigma}^{-1} \boldsymbol{\mu}+\lambda_{h} \boldsymbol{\Sigma}^{-1} \boldsymbol{\gamma} \\
\lambda_{m}=-\frac{V_{W}}{W V_{W W}}, \quad \lambda_{h}=-\frac{V_{Z W}}{W V_{W W}} .
\end{gathered}
$$

In general, $\mathbf{w}_{d}$ depends on $(W, Z, t)$ but the standard choice of $u(\cdot)$ in the literature is the CRRA type, which makes $\mathbf{w}_{d}$ depend only on $(Z, t)$. We have found that the portfolio strategy is the sum of two components. The first component is similar to the one-period solution (5) that we find in mean-variance analysis. $\boldsymbol{\Sigma}^{-1} \boldsymbol{\mu}$ defines a mean-variance portfolio and $\lambda_{m}>0$ is related to relative risk tolerance. This component will be called myopic. The second component hedges against changing investment opportunities. $\boldsymbol{\Sigma}^{-1} \boldsymbol{\gamma}$ is the portfolio with maximum correlation with the state variable, a least squares regression, and $\lambda_{h}$ is a measure of aversion to changes in the state variable with the same sign as $V_{Z W}$. This component will be called intertemporal hedging demand.

\footnotetext{
${ }^{12}$ Putting together the Bellman principle and Itô's lemma, the optimal choice is characterized by

$$
V_{W} \boldsymbol{\mu}+W V_{W W} \boldsymbol{\Sigma}_{\mathbf{w}_{d}}+V_{W Z} \gamma=0,
$$

plus the corresponding boundary condition given by $u(W(T))$ in our case.
} 
This structure ${ }^{13}$ is not far from the optimal portfolio in (10), where background risks introduced a hedging component. Now there is an intertemporal risk instead since we care about the future investment opportunities after the current period. If $V_{Z W}<0$ we interpret that higher $Z$ describes better investment opportunities (a decrease in $V_{W}$ ). For instance, let us think of such $Z$ as the short-term interest rate. In that context, there is an incentive to buy bonds because $\gamma<0$. Their price is relatively higher when it is relatively more valuable, a scenario of low interest rates. Therefore, we find a hedging motive to hold bonds that is missing in the traditional mean-variance approach.

The literature describes (13) as SAA and its myopic component as TAA since Brennan et al. (1997), which does not coincide with the industry use of those terms. It is very relevant to study cases where the optimal dynamic strategy is equal to the myopic strategy, i.e. where we find a zero hedging component. A first case is i.i.d. returns ${ }^{14}$, i.e. $\left(r_{0}, \boldsymbol{\mu}, \boldsymbol{\Sigma}\right)$ do not depend on a stochastic $Z$. But we already commented that this case does not fit empirical evidence. A context with similar implications is simply that shocks to investment opportunities cannot be hedged with available assets, $\boldsymbol{\gamma}=\mathbf{0}$. A second case is vN-M log-utility $u(W)=\ln W$ because then there is no effect of $Z$ on marginal utility of wealth, $V_{Z W}=0$. This is the growth-optimal portfolio context, which we commented above and pointed out that represents a very particular type of preferences.

On the other hand, the relevance of the intertemporal hedging will depend on the utility function and the return properties, like the persistence in $Z$. Markowitz and van Dijk (2003) show an example in discrete time where a simple rule inspired in mean-variance analysis might perform closely to an optimal dynamic strategy, which is much more difficult to compute. De Miguel et al. (2005) face the out-of-sample performance of a very simple rule, the "naive" diversification rule $\mathbf{w}=N^{-1} \mathbf{1}$ (with and without rebalancing), against several static and dynamic models, most of them commented in this survey. They study different data sets of stocks and bonds and conclude that the simple rule is not inefficient, e.g. it generally delivers the highest Sharpe ratio.

\footnotetext{
${ }^{13}$ Note that $(13)$ is still an implicit solution. We must solve a second-order partial differential equation in $V$ to compute the explicit solution, with analytic solution in few cases. Merton (1971) gives solutions for HARA utility and i.i.d. returns. Kim and Omberg (1996) and Wachter (2002) study some other few cases with closedform solutions. The former introduce mean-reversion in the drift of a single risky asset, while the latter adds consumption to that context. The addition of consumption requires the assumption of complete markets to get a closed-from solution using the approach in Cox and Huang (1989).

${ }^{14}$ In discrete time, this case also requires the assumption of CRRA utility.
} 
The length of the estimation window needs to be quite big to alleviate the estimation error and improve with respect to the simple rule.

Finally, let us describe the equilibrium implications of this set-up. They were studied in Merton (1973) and the main point is the breakdown of two-fund separation. There is three-fund separation in this context since we need a position in cash, the fund given by $\boldsymbol{\Sigma}^{-1} \boldsymbol{\mu}$ and the fund given by $\boldsymbol{\Sigma}^{-1} \boldsymbol{\gamma}$ to replicate any $\mathbf{w}_{d}$. In general, if there are $K$ state variables then we need $K+2$ funds to replicate the optimal portfolios.

Therefore, the portfolio advice cannot be simply holding the market and there is not a single optimal mix of risky assets. However, return dynamics are not interpreted as inefficiencies or deviations from equilibrium since they are part of the equilibrium. The corresponding portfolio advice represents a different interpretation of market timing. It is not active management as "beating the market" through superior information, but neither passive management as "holding the market". Similarly, the asset pricing model cannot only rely on the market portfolio, which is not necessarily mean-variance efficient. The corresponding multifactor asset pricing model is called the Intertemporal CAPM (ICAPM). On the other hand, under any of the (implausible) commented cases that imply zero hedging demands, the traditional CAPM would hold.

\subsection{Applications of Dynamic Asset Allocation}

This section and the next one describe papers that apply and extend the set-up in Section 6.2. This is one of the areas in finance with a stronger research effort during the last years, mainly spurred by the empirical evidence on predictability developed during the nineties (and the required improvement in computer power). The investment industry will soon become familiar with this approach to dynamic asset allocation.

Brennan et al. (1997) is one of the first applications of Merton (1971), solving numerically a model with utility on final wealth. The asset classes are stocks, bonds, and cash and three state variables (short-term interest rate, bond yield, and divided yield) drive time-variation in expected returns. They compare three strategies to show the difference between SAA and TAA, one with a fixed horizon of 20 years, another with a fixed horizon of 1 month, and a third one with an horizon given by a fixed date. The first one always invests more in stocks than the second one because of mean-reversion, ${ }^{15}$ while the second generally invests more in cash than

\footnotetext{
${ }^{15}$ When describing the sign of the hedging demand in this and the next applications, it is assumed that relative risk aversion is higher than one, i.e. we think of an investor that is more risk averse than a log-utility agent.
} 
the first one because of reinvestment risk. The third strategy converges from the first to the second one.

Campbell and Viceira (2001) explore the commented idea of bonds as a hedging instruments of future interest rates. They study long-term portfolio choice in an infinite horizon discrete-time model with a single source of time-variation in investment opportunities, the short-term interest rate. They use an approximate solution to the portfolio/consumption problem, which is exact under continuous time and unitary elasticity of intertemporal substitution. The portfolio of risky assets is constant over time but different from a myopic portfolio, with a positive hedging demand for bonds. The optimal ratio of bonds/stocks increases with risk aversion, which fits the professional advice and cannot be found in the traditional mean-variance analysis.

Campbell et al. (2003) apply this type of approximate solution to a discrete-time model where risk premia are also stochastic and the optimal portfolio is an affine function of state variables. They analyze another conventional advice, long-term investment in stocks because in that context stocks are relatively safer. Here the justification is that stocks can serve as a hedging instrument of their future risk premia. In a VAR equation for stocks, the dividend yield coefficient is positive and hence higher dividend yield implies higher risk premia, i.e. better investment opportunities or $V_{Z W}<0$ in our previous notation. On the other hand, the covariance between the residuals in stock and dividend yield VAR equations is negative, which the authors interpret as mean-reversion in stocks and means $\gamma<0$ in our previous notation. Another reference that explores the portfolio implications of stock predictability is Lynch (2001), but he does not treat stocks as a single asset class and works with equity portfolios formed on characteristics such as size and book to market. He shows the interaction between predictability and equity characteristics in the optimal strategy.

We have commented intertemporal hedging demands derived from time-variation in interest rates and risk premia, but volatility and learning are other relevant sources of time-variation in investment opportunities. Chacko and Viceira (2005) analyzed the first case in a continuos-time model of portfolio/consumption choice. The precision of stock returns is mean-reverting and higher volatility denotes worse investment opportunities $\left(V_{Z W}>0\right)$ while stocks show a leverage effect $(\gamma<0)$. Hence there is a negative hedging demand for stocks, but less important than the ones derived from interest rates and risk premia since volatility is not so variable and persistent.

Parameter uncertainty also introduces a hedging demand through learning. The current 
estimates of the relevant parameters become state variables and the investor has incentives to hedge their time-variation. Williams (1977) introduced heterogeneity in believes about drifts in Merton (1973) with i.i.d. returns. More recently, Brennan (1998) studies the case of learning about the risk premium of a single risky asset in a continuous-time model without predictability and consumption. A higher risk premium denotes better investment opportunities $\left(V_{Z W}<0\right)$ while Bayesian learning about the risk premium implies $\gamma>0$. Hence there is a negative hedging demand due to learning about the risk premium. Xia (2001) adds stock predictability and consumption to the previous set-up. She finds a state-dependent relationship between investment in stocks and the horizon and a non-monotone relationship between the position in stocks and the predictor.

\subsection{Link to Previous Sections}

The final part of this survey is dedicated to papers that study issues such as predictability, dynamic asset allocation, etc. in the set-up of previous sections. We will start with Bayesian portfolio choice given the connection with our last point on hedging demands from learning. Then we will explain non-normal return models and finally background risks.

- Bayesian portfolio choice: Predictability was missing in Section 4, where we generally assumed i.i.d. returns. Kandel and Stambaugh (1996) use the asset allocation of a myopic Bayesian investor to study the relevance of stock predictability. They conclude that predictability is relevant even though its statistical evidence through regressions of stock returns on predictors is weak. Barberis (2000) analyzes long-term portfolio choice by a Bayesian investor who has access to cash and stocks and uses the dividend yield as a predictor. First, he studies the case of buy-and-hold strategies under both i.i.d. and predictable returns. He finds that the investor holds less stocks the higher horizon, i.e. there are horizon effects for i.i.d. returns in a Bayesian set-up. He studies dynamic strategies and learning too. Avramov (2002) uses Bayesian model averaging to deal jointly with parameter and model uncertainty, where the latter refers to the relevant predictors of stock returns . He applies the model to buy-and-hold strategies of stock portfolios at different horizons. More recently, Hoevenaars et al. (2006) also study buy-and-hold strategies and predictability under a Bayesian (and robust) perspective.

- Nonparametric portfolio estimation: There is an alternative approach to parameter and 
model uncertainty that relies on classic inference and is developed in Brandt (1999). He proposes a kernel estimation of the portfolio/consumption choice in a discrete time model as a function of predictors. Ahït-Sahalia and Brandt (2001) adapt the previous set-up to a context with many predictors, and also take into account both expected and non-expected utility criteria. They build an optimal index that combines the different predictors and study their relative importance.

- Markov-switching models: The empirical evidence against normal returns does not mean we should forget about normal distributions since we can still work with a mixture of normal distributions. We can think of "bad times" as a low probability regime of higher volatility, lower mean, and higher correlation than "good times". Markov-switching models add persistence in the regimes (as it is the case with business cycles) and hence time-variation in investment opportunities. Ang and Bekaert (2002) show gains from international diversification in dynamic asset allocation even though there is a higher correlation between international stock markets during highly volatile bear markets. They also take into account parameter uncertainty and construct tests on portfolio weights by means of classic inference, e.g. they do not find significant intertemporal hedging. In a similar spirit, Ang and Bekaert (2004) develop other applications but the portfolio strategy is simplified to switching between two mean-variance choices. Guidolin and Timmermann (2005a) estimate four different regimes in a VAR model with large and small stocks, bonds, and cash. There is not a monotone relationship between the stock position and the horizon due to the interaction between learning and predictability in a dynamic portfolio choice based on final wealth. Guidolin and Timmermann (2005b) use two regimes and preferences defined on the first four moments of wealth. Both features can justify the home bias of a US investor.

- Poisson processes: Section 6.2 was meant for diffusions and another modelling device of non-normal returns is the introduction of Poisson processes to model jumps in continuos time. Merton (1971) already did it in different contexts such as the price of a bond. More recently, Liu et al. (2003) study dynamic asset allocation with utility from final wealth when there are jumps in both prices and volatility of a single risky asset. They compute an analytical solution where the desire to hold leveraged or short positions decreases with respect to the diffusion case. In fact, the optimal strategy can be interpreted as a mixture 
between a dynamic and a buy-and-hold strategy, as if some component of wealth was illiquid.

- Inflation: Brennan and Xia (2002) focus on portfolio/consumption choice with nominal assets under inflation. They use a continuous time model where the investor has access to cash, stocks and bonds with different maturities. Risk premia are constant but the short-term interest rate and inflation are predictable. They show how horizon and risk aversion affect the optimal mix of stocks and bonds and also the chosen bond maturity under short-sale constraints.

- Human capital: There is a brief comment on the impact of wages in Merton (1971). More recently, The impact of human capital in portfolio/consumption choice is studied in Koo (1998) and Viceira (2001). The former uses a continuous time model with several risky securities, while the latter uses a discrete time model with uncertainty about retirement and death and a single risky security interpreted as stocks. In both papers, human capital is exogenous and nontradable, and investment opportunities in financial markets are i.i.d. Bodie et al. (1992) explore the impact of labor supply flexibility in a continuous time model.

- Housing: Real estate can be traded but its liquidity is much lower than financial assets. Portfolio choice and asset pricing with an illiquid durable consumption good, that we can interpret as housing, was analyzed in a continuous time model by Grossman and Laroque (1990). More recent references on the interaction between housing and portfolio/consumption choice are Cocco (2005) and Yao and Zhang (2005). They develop discrete time models that also take into account (exogenous and nontradable) human capital. On the other hand, they only analyze stocks and cash as asset classes and assume that investment opportunities in financial markets are i.i.d.

- ALM: We also pointed out that pension funds' ALM has an important dynamic component. We refer the interested reader to Ziemba and Mulvey (1998) and Scherer (2003) for details. For instance, in former reference's Chapter 16, R.C. Merton applies the benchmark model of Section 6.2 to university endowment funds, computing the optimal portfolio strategy that takes into account that the university has other (tangible and intangible) assets. 


\section{References}

Ahït-Sahalia, and M.W. Brandt (2001): Variable Selection and Portoflio Choice, Journal of Finance 56, 1297-1351.

Aït-Sahalia, Y., P.A. Mykland, and L. Zhang (2005): How Often to Sample a ContinuousTime Process in the Presence of Market Microstructure Noise, Review of Financial Studies 18, $351-416$.

Alexander, G.J., and A.M. Baptista (2002): Economic Implications of Using a Mean-VaR Model for Portfolio Selection: A Comparison with Mean-Variance Analysis, Journal of Economic Dynamics and Control 26, 1159-1193

Ang, A., and G. Bekaert (2002): International Asset Allocation with Regime Shifts, Review of Financial Studies 15, 1137-1187.

Ang, A., and G. Bekaert (2004): How Regimes Affect Asset Allocation?, Financial Analysts Journal 60, March/April.

Ang, A., and J. Chen (2002): Asymmetric Correlations of Equity Portfolios, Journal of Financial Economics 63, 443-494.

Arditti, F. and H. Levy (1976): Portfolio Efficiency Analysis in Three Moments: The Multiperiod Case, Journal of Finance 30, 797-809.

Avramov, D. (2002): Stock Return Predictability and Model Uncertainty, Journal of Financial Economics 64, 423-458.

Bai, J., and S. Ng (2005): Test for Skewness, Kurtosis, and Normality for Time Series Data, Journal of Business and Economic Statistics 23, 49-61.

Barberis, N. (2000): Investing in the Long Run when Returns are Predictable, Journal of Finance 55, 225-264.

Barry, C.B. (1974): Portfolio Analysis Under Uncertain Means, Variances, and Covariances, Journal of Finance 29, 515-522.

Barry, C.B., and S.J. Brown (1985): Differential Information and Security Market Equilibrium, Journal of Financial and Quantitative Analysis 20, 407-422.

Bawa, V. (1978): Safety First, Stochastic Dominance and Optimal Portfolio Choice, Journal of Financial and Quantitative Analysis 13, 255-271.

Bawa, V., and E. Lindenberg (1977): Capital Market Equilibrium in a Mean-Lower Partial Moment Framework, Journal of Financial Economics 5, 189-200. 
Bekaert, G., C.B. Erb, C.R. Harvey, and T.E. Viskanta (1998): Distributional Characteristics of Emerging Market Returns and Asset Allocation, Journal of Portfolio Management 24, Winter.

Berk, J.B. (1997): Necessary Conditions for the CAPM, Journal of Economic Theory 73, 245-257.

Best, M.J. and R.R. Grauer (1991): On the Sensitivity of Mean-Variance-Efficient Portfolios to Changes in Asset Means: Some Analyitical and Computational Results, The Review of Financial Studies 4, 315-342.

Black, F. (1972): Capital Market Equilibrium with Restricted Borrowing, Journal of Business 45, 444-455.

Black, F. and R. Litterman (1992): Global Portfolio Optimization, Financial Analysts Journal 48, September-October.

Bodie, Z., R.C. Merton, and W.F. Samuelson (1992): Labor Supply Flexibility and Portfolio Choice in a Life Cycle Model, Journal of Economic Dynamics and Control 16, 427-449.

Brandt, M.W. (1999): Estimating Portfolio and Consumption Choice: A Conditional Euler Equations Approach, Journal of Finance 54, 1609-1645.

Brandt, .M W. (2004): Portfolio Choice Problems, in Y. Ait-Sahalia and L.P. Hansen (eds.), Handbook of Financial Econometrics, forthcoming.

Brennan, M.J. (1998): The Role of Learning in Dynamic Portfolio Decisions, European Finance Review 1, 295-306.

Brennan, M.J., E.S. Schwartz, and R. Lagnado (1997): Strategic Asset Allocation, Journal of Economic Dynamics and Control 21, 1377-1403.

Brennan, M.J., and Y. Xia (2002): Dynamic Asset Allocation under Inflation, Journal of Finance 57, 1201-1238.

Brinson, G.P., L.R. Hood, and G.L. Beebower (1986): Determinants of Portfolio Performance, Financial Analysts Journal 42, July/August.

Brito, N.O. (1977): Marketability Restrictions and the Valuation of Capital Assets Under Uncertainty, Journal of Finance 32, 1109-1123.

Britten-Jones, M. (1999): The Sampling Error in Estimates of Mean-Variance Efficient Portfolio Weights, Journal of Finance 54, 655-671.

Brunnermeier, M.K. (2001): "Asset Pricing under Asymmetric Information", Oxford University Press. 
Campbell, J.Y., Y.L. Chan, and L.M. Viceira (2003): A Multivariate Model of Strategic Asset Allocation, Journal of Financial Economics 67, 41-80.

Campbell, J.Y, A.W. Lo, and A.C. McKinlay (1997): "The Econometrics of Financial Markets", Princeton University Press.

Campbell, J.Y., and L.M. Viceira (2001): Who Should Buy Long-Term Bonds?, American Economic Review 91, 99-127.

Campbell, J.Y., and L.M. Viceira (2002): "Strategic Asset Allocation: Portfolio Choice for Long-Term Investors", Oxford University Press.

Campbell, J.Y., and L.M. Viceira (2005): The Term Structure of the Risk-Return Tradeoff, Financial Analysts Journal 61, January/February.

Canner, N., N.G. Mankiw, and D.N. Weil (1997): An Asset Allocation Puzzle, American Economic Review 87, 181-191.

Chacko, G., and L.M. Viceira (2005): Dynamic Consumption and Portfolio Choice with Stochastic Volatility in Incomplete Markets, Review of Financial Studies 18, 1369-1402.

Chamberlain, G. (1983): A Characterization of the Distributions that Imply Mean-Variance Utility Functions, Journal of Economic Theory 29, 185-201.

Chamberlain, G. and M. Rothschild (1983): Arbitrage, Factor Structure, and Mean-Variance Analysis on Large Asset Markets, Econometrica 51, 1281-1304.

Chan, L.K.C., J. Karceski, and J. Lakonishok (1999): On Portfolio Optimization: Forecasting Covariances and Choosing the Risk Model, Review of Financial Studies 12, 937-974.

Chopra, V.K., and W.T. Ziemba (1993): The Effect of Errors in Means, Variances, and Covariances on Optimal Portfolio Choice, Journal of Portfolio Management 19, 6-11.

Clarke, R.C. and H. de Silva (1998): State-Dependent Asset Allocation, Journal of Portfolio Management 24, Winter.

Cocco, J.F. (2005): Portfolio Choice in the Presence Housing, Review of Financial Studies $18,535-567$.

Cochrane, J.H. (2001): “Asset Pricing", Princeton University Press.

Cox, J., and C.-F. Huang (1989): Optimal consumption and Portfolio Policies When Asset Prices Follow a Diffusion Process, Journal of Economic Theory 49, 33-83.

Daníelsson, J., B.N. Jorgensen, M. Sarma, C.G. de Vries, and J.-P. Zigrand (2006): Consistent Measures of Risk, FMG DP 565. 
De Athayde, G.M., and R.G. Flôres (2004): Finding a Maximum Skewness PortfolioA General Solution to Three-Moments Portfolio Choice, Journal of Economic Dynamics and Control 28, 1335-1352.

De Miguel, V., L. Garlappi, and R. Uppal (2005): How Inefficient is the 1/N Asset-Allocation Strategy?, forthcoming Review of Financial Studies.

Eling, M., and F. Schuhmacher (2007): Does the Choice of Performance Measure Influence the Evaluation of Hedge Funds?, forthcoming Journal of Banking and Finance.

Ferson, W.E., and A.F. Siegel (2001): The Efficient Use of Conditioning Information in Portfolios, Journal of Finance 56, 967-982.

Friend, I., Y. Landskroner, and E. Losq (1976): The Demand for Risky Assets Under Uncertain Inflation, Journal of Finance 31, 1287-1297.

Frost, P.A., and J.E. Savarino (1986): An Empirical Bayes Approach to Efficient Portfolio Selection, Journal of Financial and Quantitative Analysis 21, 293-305.

Frost, P.A., and J.E. Savarino (1988): For Better Performance: Constrain Portfolio Weights, Journal of Portfolio Management 15, 29-34.

Fung, W., and D.A. Hsieh (1999): Is Mean-Variance Applicable to Hedge Funds?, Economic Letters 62, 53-58.

Garlappi, L., R. Uppal, and T. Wang (2007): Portfolio Selection with Parameter and Model Uncertainty: A Multi-Prior Approach, Review of Financial Studies 20, 41-81.

Geweke, J. (2001): A Note on Some Limitations of CRRA Utility, Economic Letters 71, 341-345.

Gonedes, N.J. (1976): Capital Market Equilibrium for a Class of Heterogeneous Expectations in a Two-Parameter World, Journal of Finance 31, 1-15.

Grinold, R.C., and R.N. Kahn (1999): “Active Portfolio Management”, McGraw-Hill.

Grossman, S.J., and G. Laroque (1990): Asset Pricing and Optimal Portfolio Choice in the Presence of Illiquid Durable Consumption Goods, Econometrica 58, 25-51.

Guidolin, M., and A. Timmermann (2005a): Asset Allocation under Multivariate Regime Switching, FRB of St. Louis Working Paper 2005-002C.

Guidolin, M., and A. Timmermann (2005b): International Asset Allocation under Regime Switching, Skew and Kurtosis Preferences, FRB of St. Louis Working Paper 2005-034B. 
Guidolin, M. and A. Timmermann (2006): Term Structure of Risk under Alternative Econometric Specifications, Journal of Econometrics 131, 285-308.

Hansen, L.P., and S.F. Richard (1987): The Role of Conditioning Information in Deducing Testable Restrictions Implied by Dynamic Asset Pricing Models, Econometrica 55, 587-613.

Harlow, W. (1991): Asset Allocation in a Downside Risk Framework, Financial Analyst Journal 47, Sept/Oct.

Harlow, W., and R. Rao (1989): Asset Pricing in a Generalised Mean-Lower Partial Moment Framework: Theory and Evidence, Journal of Financial and Quantitative Analysis 24, 285-311.

Harvey, C.R., J.C. Liechty, M.W. Liechty, and P. Müller (2004): Portfolio Selection with Higher Moments, Duke University FRPS04-123.

Harvey, C.R., and A. Siddique (2000): Conditional Skewness in Asset Pricing Tests, Journal of Finance 55, 1263-1295.

He, G., and R. Litterman (1999): The Intuition Behind Black-Litterman Model Portfolios, Goldman Sachs Investment Management Series.

Hlawitschka, W. (1994): The Empirical Nature of Taylor-Series Approximations to Expected Utility, American Economic Review 84, 713-719.

Hodges, C.W., W.R.L. Taylor, and J.A. Yoder (1997): Stocks, Bonds, the Sharpe Ratio, and the Investment Horizon, Financial Analysts Journal 53, Nov/Dec.

Hoevenaars, R., R. Molenaar, P. Schotman, and T. Steenkamp (2006): Strategic Asset Allocation for Long-Term Investors: Parameter Uncertainty and Prior Information, LIFE WP06-003.

Hogan, W.W., and J.M. Warren (1972): Computation of the Efficient Boundary in the E-S Portfolio Selection Model, Journal of Financial and Quantitative Analysis 7, 1881-1896.

Hogan, W.W. and J.M. Warren (1974): Toward the Development of an Equilibrium Based Capital Market Model Based on Semivariance, Journal of Financial and Quantitative Analysis $9,1-11$.

Horowitz, J.L. (2001): The Bootstrap, in J.J. Heckman and E.E. Leamer (eds.), Handbook of Econometrics vol. 5, Elsevier Science Publishers.

Huang, C. and R. Litzenberger (1988): "Foundations for Financial Economics", NorthHolland, New-York.

Ingersoll, J.E. (1975): Multidimensional Security Pricing, Journal of Financial and Quantitative Analysis 10, 785-798. 
Ingersoll, J.E. (1987): “Theory of Financial Decision Making”, Rowman \& Littlefield.

Jagannathan, R., and T. Ma (2003): Risk Reduction in Large Portfolios: Why Imposing the Wrong Constraints Helps, Journal of Finance 58, 1651-1684.

Jobson, J.D., and B. Korkie (1980): Estimation for Markowitz Efficient Portfolios, Journal of the American Statistical Association 75, 544-554.

Jobson, J.D., and B. Korkie (1983): Statistical Inference in Two-Parameter Portfolio Theory with Multiple Regression Software, Journal of Financial and Quantitative Analysis 18, 189-197.

Jondeau, E., and M. Rockinger (2006): Optimal Portfolio Allocation under Higher Moments, European Financial Management 12, 29-55.

Jorion, P. (1986): Bayes-Stein Estimation for Portfolio Analysis, Journal of Financial and Quantitative Analysis 21, 278-292.

Jorion, P. (1992): Portfolio Optimization in Practice, Financial Analysts Journal 48, Jan/Feb.

Kandel, S., and R.F. Stambaugh (1996): On the Predictability of Stock Returns: An AssetAllocation Perspective, Journal of Finance 51, 385-424.

Keating, C., and F. Shadwick (2002): A Universal Performance Measure, Journal of Performance Measurement 6, 59-84.

Kim, T.S., and E. Omberg (1996): Dynamic Nonmyopic Portfolio Behavior, Review of Financial Studies 9, 141-161.

Klein, R.W., and V.S. Bawa (1976): The Effect of Estimation Risk on Optimal Portfolio Choice, Journal of Financial Economics 3, 215-231.

Koo, H.K. (1998): Consumption and Portfolio Selection with Labor Income: A Continuous Time Approach, Mathematical Finance 8, 49-65.

Krawss, A., and R. Litzenberger (1976): Skewness Preference and the Valuation of Risky Assets, Journal of Finance 31, 1085-1100.

Kroll, Y., H. Levy, and H.M. Markowitz (1984): Mean-Variance Versus Direct Utility Maximization, Journal of Finance 39, 47-61.

Ledoit, O., and M. Wolf (2003): Improved Estimation of the Covariance Matrix of Stock Returns With an Application to Portfolio Selection, Journal of Empirical Finance 10, 603-621.

Lee, W. (2001): “Theory and Methodology of Tactical Asset Allocation”, John Wiley \& Sons Inc. 
Levy, H., and R. Duchin (2004): Asset Return Distributions and the Investment Horizon, Journal of Portfolio Management 30, Spring.

Levy, H., and H.M. Markowitz (1979): Approximating Expected Utility by a Function of Mean and Variance, American Economic Review 69, 308-317.

Lintner, J. (1965): The Valuation of Risk Assets and the Selection of Risky Investments in Stock Portfolios and Capital Budgets, Review of Economics and Statistics 47, 13-37.

Lintner, J. (1969): The Aggregation of Investor's Diverse Judgments and Preferences in Purely Competitive Security Markets, Journal of Financial and Quantitative Analysis 4, 347400.

Litterman, B. (ed.) (2003): "Modern Investment Management: An Equilibrium Approach", Wiley.

Liu, J., F.A. Longstaff, and J. Pan (2003): Dynamic Asset Allocation with Event Risk, Journal of Finance 58, 231-259.

Lutgens, F., and P.C. Schotman (2007): Robust Portfolio Optimisation with Multiple Experts, CEPR DP 6161.

Lynch, A. (2001): Portfolio Choice and Equity Characteristics: Characterizing the Hedging Demands Induced by Return Predictability, Journal of Financial Economics 62, 67-130.

Markowitz, H. (1952): Portfolio Selection, Journal of Finance 7, 77-99.

Markowitz, H. (1959): "Portfolio Selection: Efficient Diversification of Investments", New York: John Wiley \& Sons.

Markowitz, H., and E.L. van Dijk (2003): Single-Period Mean-Variance Analysis in a Changing World, Financial Analysts Journal 59, Mar/Apr.

Mayers, D. (1973): Nonmarketable Assets and the Determination of Capital Asset Prices in the Absence of a Riskless Asset, Journal of Business 46, 258-267.

Merton, R.C. (1969): Lifetime Portfolio Selection Under Uncertainty: The Continuous-Time Case, Review of Economics and Statistics 51, 247-257.

Merton, R.C. (1971): Optimum Consumption and Portfolio Rules in a Continuous-Time Model, Journal of Economic Theory 3, 373-413.

Merton, R.C. (1972): An Analytic Derivation of the Efficient Portfolio Frontier, Journal of Financial and Quantitative Analysis 7, 1851-1872. 
Merton, R.C. (1973): An Intertemporal Capital Asset Pricing Model, Econometrica 41, $867-887$.

Merton, R.C. (1980): On Estimating the Expected Return on the Market: An Exploratory Investigation, Journal of Financial Economics 8, 323-361.

Merton, R.C. (2001): “Continuous Time Finance”, Blackwell.

Michaud, R. (1989): The Markowitz Optimization Enigma: Is Optimized Optimal?, Financial Analysts Journal 45, Jan/Feb.

Michaud, R.O. (1998): "Efficient Asset Management", Oxford University Press.

Mossin, J. (1966): Equilibrium in a Capital Asset Market, Econometrica 34, 768-783.

Mossin, J. (1968): Optimal Multiperiod Portfolio Choices, Journal of Business 41, 215-229.

Owen, J., and R. Rabinovitch (1983): On the Class of Elliptical Distributions and their Applications to the Theory of Portfolio Choice, Journal of Finance 58, 745-752.

Pástor, L. (2000): Portfolio Selection and Asset Pricing Models, Journal of Finance 55, $179-223$.

Pástor, L. and R.F. Stambaugh (2000): Comparing Asset Pricing Models: An Investment Perspective, Journal of Financial Economics 56, 335-381.

Pedersen, C.S., and S.E. Satchell (2002): On the Foundation of Performance Measure under Asymmetric Returns, Quantitative Finance 2, 217-223.

Peiró, A. (1999): Skewness in Financial Returns, Journal of Banking and Finance 23, 847862.

Qian, E., and S. Gorman (2001): Conditional Distribution in Portfolio Theory, Financial Analysts Journal 57, March/April.

Roll, R. (1977): A Critique of the Asset Pricing Theory's Tests Part I: On Past and Potential Testability of the Theory, Journal of Financial Economics 4, 129-176.

Roll, R. (1992): A Mean-Variance Analysis of Tracking Error, Journal of Portfolio Management 18, 13-22.

Roy, A. (1952): Safety First and the Holding of Assets, Econometrica 20, 431-439.

Rubinstein, M. (1973): The Fundamental Theorem of Parameter-Preference Security Valuation, Journal of Financial and Quantitative Analysis 8, 61-69.

Samuelson, P. (1969): Lifetime Portfolio Selection bt Dynamic Stochastic Programming, Review of Economics and Statistics 51, 239-246. 
Sánchez-Torres, P.L., and E. Sentana (1998): Mean-Variance-Skewness Analysis: An Application to Risk Premia in the Spanish Stock Market, Investigaciones Económicas 22, 5-17.

Scherer, B. (2002): Portfolio Resampling: Review and Critique, Financial Analysts Journal 58 , Nov/Dec.

Scherer, B. (ed.) (2003): “Asset and Liability Management Tools: A Handbook for Best Practice", Risk Books.

Scherer, B. (2004): "Portfolio Construction and Risk Budgeting", Risk Books.

Sentana, E. (2005): Least Squares Predictions and Mean-Variance Analysis, Journal of Financial Econometrics 3, 56-78.

Sharpe, W.F. (1963): Mutual Fund Performance, Journal of Business 39, 119-138.

Sharpe, W.F. (1964): Capital Asset Prices: A Theory of Market Equilibrium under Conditions of Risk, Journal of Finance 19, 425-442.

Sharpe, W.F. (1987): Integrated Asset Allocation, Financial Analysts Journal 43, Sep/Oct.

Sharpe, W.F. (1994): The Sharpe Ratio, Journal of Portfolio Management Fall, 49-58.

Sharpe, W.F., and L.G. Tint (1990): Liabilities - A New Approach, Journal of Portfolio Management 16, Winter.

Simaan, Y. (1993): Portfolio Selection and Capital Asset Pricing -Three Parameter Framework, Management Science 39, 568-577.

Solnik, B. (1978): Inflation and Optimal Portfolio Choices, Journal of Financial and Quantitative Analysis 13, 903-925.

Sortino, F. and H. Forsey (1996): On the Use and Misuse of Downside Risk, Journal of Portfolio Management 22, Winter.

Stevens, G.V.G. (1998): On the Inverse of the Covariance Matrix in Portfolio Analysis, Journal of Finance53, 1821-1827.

Stutzer, M. (2000): A Portfolio Performance Index, Financial Analysts Journal 56, May/June.

Stutzer, M. (2003): Portfolio Choice with Endogenous Utility: A Large Deviations Approach, Journal of Econometrics 116, 365-386.

Tobin, J. (1958): Liquidity Preference as Behavior Toward Risk, Review of Economic Studies $25,65-86$.

Tu, J., and G. Zhou (2004): Data-Generating Process Uncertainty: What Difference Does it Make in Portfolio Decisions?, Journal of Financial Economics 72, 385-421. 
Viceira, L.M. (2001): Optimal Portfolio Choice for Long-Horizon Investors with Nontradable Labor Income, Journal of Finance 56, 433-470.

Wachter, J.A. (2002): Portfolio and Consumption Decisions under Mean-Reverting Returns: An Exact Solution for Complete Markets, Journal of Financial and Quantitative Analysis 37, $63-91$.

Williams, J.T. (1977): Capital Asset Prices with Heterogeneous Beliefs, Journal of Financial Economics 5, 219-239.

Xia, Y. (2001): Learning about Predictability: The Effects of Parameter Uncertainty on Dynamic Asset Allocation, Journal of Finance 56, 205-246.

Yao, R., and H.H. Zhang (2005): Optimal Consumption and Portfolio Choices with Risky Housing and Borrowing Constraints, Review of Financial Studies 18, 197-239.

Ziemba, W.T, and J.M. Mulvey (eds.) (1998): "Worldwide Asset and Liability Modeling", Cambridge Univeristy Press. 


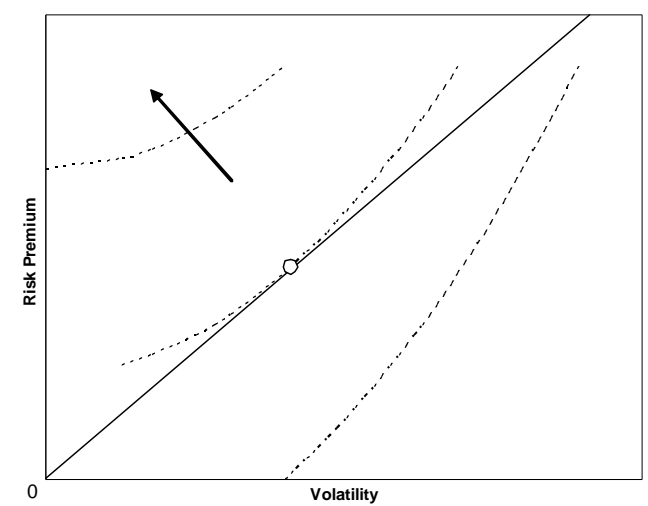

Figure 1: Mean-variance preferences and frontier.

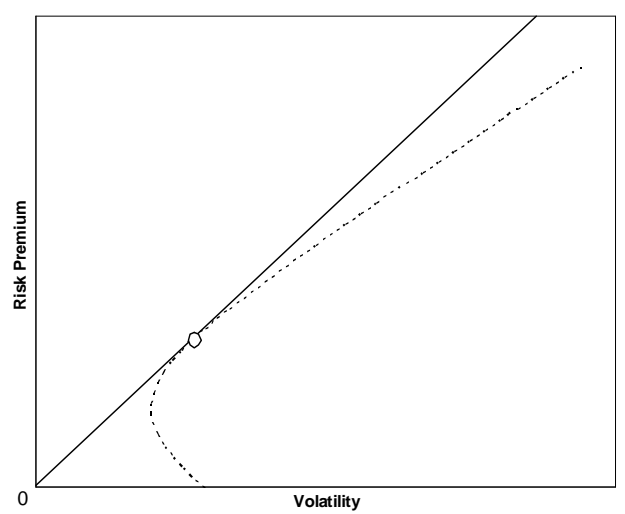

Figure 2: Mean-variance frontiers with and without a safe asset.

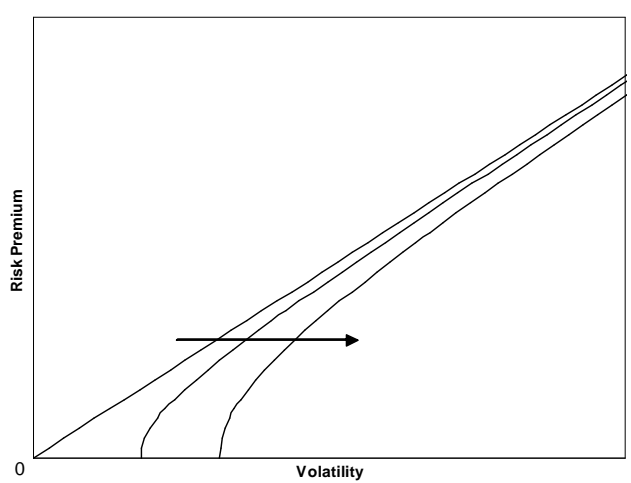

Figure 3: Mean-variance frontiers with and without background risks. 